NIST Special Publication 1173

\title{
Virtual Cement and Concrete Testing Laboratory
}

Jeffrey W. Bullard

\section{Version 9.5 User Guide}

dx.doi.org/10.6028/NIST.SP.1173

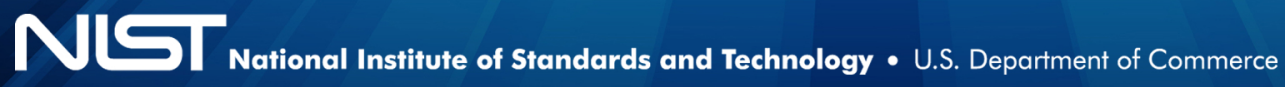




\section{Virtual Cement and Concrete Testing Laboratory}

\section{Version 9.5 User Guide}

Jeffrey W. Bullard

Materials and Structural Systems Division

Engineering Laboratory

http://dx.doi.org/10.6028/NIST.SP.1173

May 2014

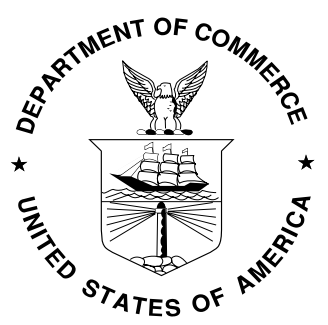

U.S. Department of Commerce Penny Pritzker, Secretary

National Institute of Standards and Technology Patrick D. Gallagher, Under Secretary of Commerce for Standards and Technology and Director 
Certain commercial entities, equipment, or materials may be identified in this document in order to describe an experimental procedure or concept adequately. Such identification is not intended to imply recommendation or endorsement by the National Institute of Standards and Technology, nor is it intended to imply that the entities, materials, or equipment are necessarily the best available for the purpose.

National Institute of Standards and Technology Special Publication 1173

Natl. Inst. Stand. Technol. Spec. Publ. 1173, 60 pages (May 2014) http://dx.doi.org/10.6028/NIST.SP.1173

CODEN: NSPUE2 


\title{
Virtual Cement and Concrete Testing Laboratory Version 9.5 User Guide
}

\author{
Jeffrey W. Bullard ${ }^{1}$ \\ Materials and Structural Systems Division \\ National Institute of Standards and Technology \\ Gaithersburg, Maryland USA 20899-8615
}

This document serves as the user's guide for the Virtual Cement and Concrete Testing Laboratory (VCCTL) software, version 9.5. Using the VCCTL software, a user may create 3D microstructures of cement paste made with portland cement clinker, calcium sulfate, fly ash, slag, limestone, and other materials. Hydration of these microstructures can be simulated under a variety of curing conditions, and the resulting hardened material can be analyzed for a number of properties including linear elastic moduli, compressive strength, and relative diffusion coefficients. A 3D packing of fine and coarse aggregates in mortar and concrete materials also can be created. The VCCTL software uses a relational database and plotting capabilities to facilitate viewing important characteristics of cement powders, supplementary cementitious materials, fillers, and aggregates.

Keywords: Building technology, cement hydration, computer modeling, concrete testing, microstructure, simulation, virtual laboratory

${ }^{1}$ Email: jeffrey.bullard@nist.gov 


\section{Contents}

1 Introduction 1

1.1 Disclaimer . . . . . . . . . . . . . . . . . 1

1.2 Intent and Use . . . . . . . . . . . . . . . . . . . . . . . 1

1.3 Notation Conventions ................. 2

2 Installation $\quad 2$

2.1 Software Prerequisites ......................... 2

2.2 Terms of Use Agreement . . . . . . . . . . . . . . . . . . 3

2.3 Installation Folder . . . . . . . . . . . . . . . . 3

2.4 Ready to Install . . . . . . . . . . . . . . . . . . . 4

2.5 Finishing the Installation . . . . . . . . . . . . . 4

3 Launching and Closing a Session 5

3.1 Closing VCCTL . . . . . . . . . . . . 5

4 Logging On $\quad 6$

5 The Main Page 6

5.1 Announcements and Tutorials ............... 6

5.2 The Menu Bar . . . . . . . . . . . . . . . . . 7

6 Lab Materials 8

6.1 Cements ...................... 8

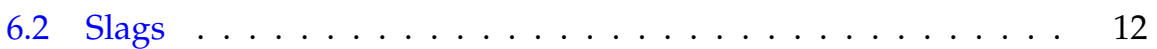

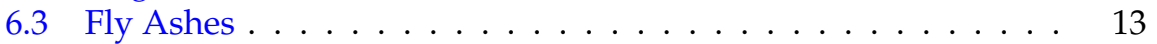

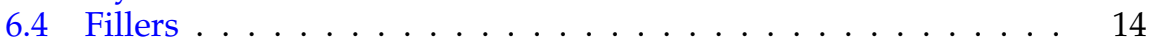

6.5 Aggregates ......................... 14

7 Mix 16

7.1 A Note on the Operating Principles of VCCTL Programs . . . 16

7.2 Prepare Mix . . . . . . . . . . . . . . . 16

7.3 Hydrate Mix . . . . . . . . . . . . . . . . 22

8 Measurements 26

8.1 Plot Hydrated Properties . . . . . . . . . . . . . . . . . . . . 27

8.2 Measure Cement/Concrete Properties . . . . . . . . . . . . . 29

9 My Operations 33

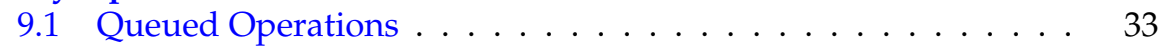

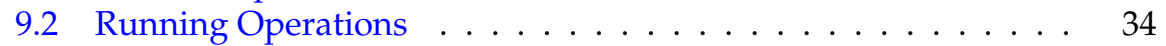

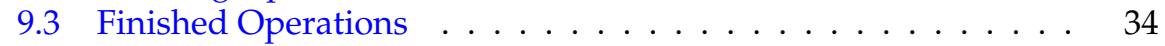

9.4 Cancelled Operations . . . . . . . . . . . . . . 35 
10 My Files $\quad 35$

10.1 Microstructure Files . . . . . . . . . . . . . . . . 35

10.2 Displaying microstructure images . . . . . . . . . . . . . . . . 38

10.3 Aggregate Files . . . . . . . . . . . . . . . . . . . . . . 40

10.4 Hydration Files . . . . . . . . . . . . . . . . . . . . . . . . . . . . . . . . 41

10.5 Mechanical Properties Files . . . . . . . . . . . . . . . . . . . 44

10.6 Transport Properties Files . . . . . . . . . . . . . . 45

11 Logout $\quad 46$

Appendix A Preparing and Uploading New Cement Data 47

A.1 Getting Ready . . . . . . . . . . . . . . . . . . . . 47

A.2 Running processImageJ . . . . . . . . . . . . . . 48

A.3 What To Do Next .................. 48

Appendix B Integer ID Numbers of VCCTL Phases 50

Appendix C Contents of Hydration Results File 51

$\begin{array}{ll}\text { References } & 53\end{array}$ 


\section{Introduction}

This document provides guidance for using the Virtual Cement and Concrete Testing Laboratory software, version 9.5. This virtual laboratory software consists of a graphical user interface and the underlying computer models and programs that allow users to create, hydrate, and estimate the performance of $3 \mathrm{D}$ cement-based microstructures from a desktop computer. The primary intent of this version is educational, especially to supplement the classroom experience of students studying civil engineering materials technology and materials science.

The main underlying programs for the VCCTL software have been described previously $[1,2,15]$, and a large number of applications of these models has been published $[3-5,7-9,12,13]$. This user guide therefore is mostly focused on providing a detailed description of the user interface.

\subsection{Disclaimer}

This software was developed at the National Institute of Standards and Technology (NIST) by employees of the Federal Government in the course of their official duties. Pursuant to Title 17 Section 105 of the United States Code this software is not subject to copyright protection and is in the public domain. The VCCTL is an experimental system. NIST assumes no responsibility whatsoever for its use by other parties, and makes no guarantees, expressed or implied, about its quality, reliability, or any other characteristic. We would appreciate acknowledgment, such as an appropriate citation in a publication, if the software is used.

The U.S. Department of Commerce makes no warranty, expressed or implied, to users of VCCTL and associated computer programs, and accepts no responsibility for its use. Users of VCCTL assume sole responsibility under Federal law for determining the appropriateness of its use in any particular application; for any conclusions drawn from the results of its use; and for any actions taken or not taken as a result of analyses performed using these tools.

Users are warned that VCCTL is intended for educational use only and is intended only to supplement the informed judgment of the user. The software package contains computer models that may or may not have predictive value when applied to a specific set of factual circumstances. Lack of accurate predictions by the models could lead to erroneous conclusions with regard to materials selection and design. All results should be evaluated by an informed user.

\subsection{Intent and Use}

The algorithms, procedures, and computer programs described in this guide constitute a prototype system for a virtual laboratory for the testing of cement and concrete. They have been compiled from the best knowledge and understanding currently available, but have important limitations that must be un- 
derstood and considered by the user. VCCTL is intended for use by persons competent in the field of cement-based materials and having some familiarity with computers.

\subsection{Notation Conventions}

Throughout this guide, the following text conventions will be used:

- italicized text will indicate

- words on a VCCTL window or computer terminal window (for example, the Save button);

- key combinations Ctrl-C to indicate the keyboard combination of Control plus C).

- Courier font will be used to indicate

- a file name: mycement.img or disrealnew.exe;

- the location of a folder or file: C: \Users \user \vcctl;

- web addresses: http://oracle.com

- Bold text will indicate an important note or warning that the user must heed (for example, Never close the command window during a VCCTL session).

- All capital letters will be used to denote variables in file names or user names for which the user must supply the correct value. For example, PATH_TO_VCCTL_INSTALL_DIRECTORY, the user is expected to substitute the correct path, such as C: \Users \bullard \Desktop\vcctl.

\section{Installation}

\subsection{Software Prerequisites}

The following requirements must be met for successful installation and running of this distribution of VCCTL:

- A personal computer running Microsoft Windows $7^{2}$ either natively or as a virtual machine. Other versions of Windows, such as XP, Vista, or Windows 8, may or may not be compatible with VCCTL.

\footnotetext{
${ }^{2}$ Certain commercial equipment and/or materials are identified in this report in order to adequately specify the experimental procedure. In no case does such identification imply recommendation or endorsement by the National Institute of Standards and Technology, nor does it imply that the equipment and/or materials used are necessarily the best available for the purpose.
} 
- A minimum of 2 GB RAM and minimum hard disk space of 20 GB for long-term use of the software. Multiprocessor architectures with expanded shared memory, such as 16 GB or more, are commonly available and are recommended for the simultaneous execution of multiple simulations without loss of performance.

- Java SE Development Kit (JDK) 7, Update 51, or a more recent update. Go to http://www .oracle.com/technetwork/java/javase/downloads/ jdk7-downloads-1880260. html to download the latest release of the JDK and follow the installation instructions given there.

- Mozilla Firefox, Google Chrome, or Apple Safari web browser. Not required for installation, but Microsoft's Internet Explorer sometimes produces erratic results when running the software.

To install VCCTL, drag the vcctl-9.5-installer-windows.exe icon to the Desktop. Once there, double-click on the installation icon. A series of windows will guide you through the installation process.

\subsection{Terms of Use Agreement}

This dialog requires you to acknowledge the software disclaimer. If you accept the disclaimer agreement, you proceed with the installation by pressing the Next button. Otherwise, you will not be able to install and use the VCCTL software.

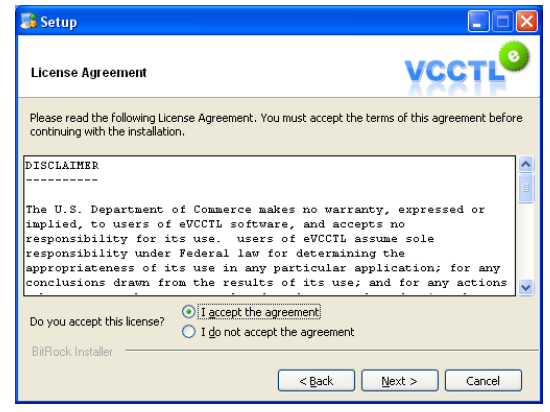

\subsection{Installation Folder}

You may install VCCTL in any location on your computer, provided that you have the adequate read/write permissions. The default path and folder is vcctl located in your user folder. You may accept that default or change it to another location. However, the final folder on the path must be named vcctl in all lowercase letters. The software will not work properly if it is not installed in a folder named vcctl. 


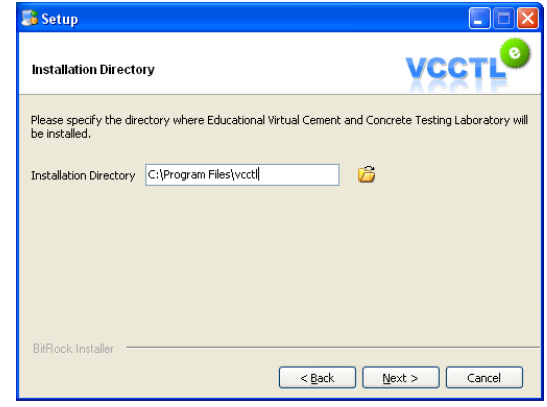

\subsection{Ready to Install}

The next dialog box indicates that the software is ready to install. Click the Next button to begin the installation.

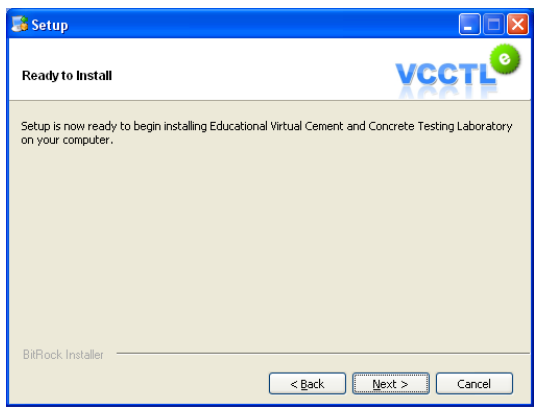

The last step of the installation is to unpack a rather large data folder. This step can consume several minutes, during which time it will appear that nothing is happening. Please be patient while the data folder unpacks.

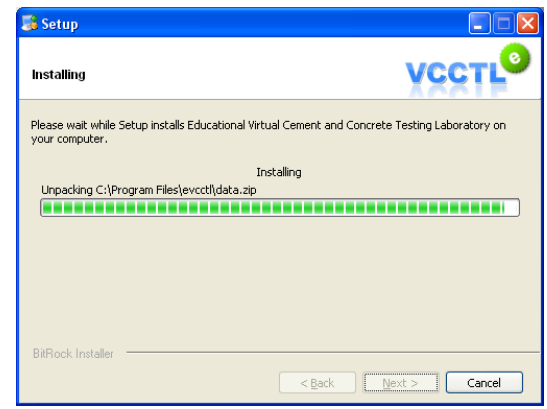

\subsection{Finishing the Installation}

When the final step is completed, a final dialog box will appear. Close this box by clicking on the Finish button. 


\section{Launching and Closing a Session}

Before running VCCTL for the first time, it is helpful to make

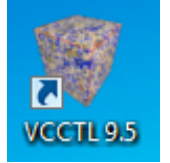

Mozilla Firefox your default web browser. To do this, open Mozilla Firefox. If it is not already your default browser, a dialog box should appear asking you if you want to make it your default browser. Click Yes and close the browser after it opens. If the installation was successful, VCCTL can be launched from the Desktop by double-clicking on the Desktop icon labeled VCCTL 9.5.

A Java console window will appear on the desktop, with a lot of information scrolling through it:

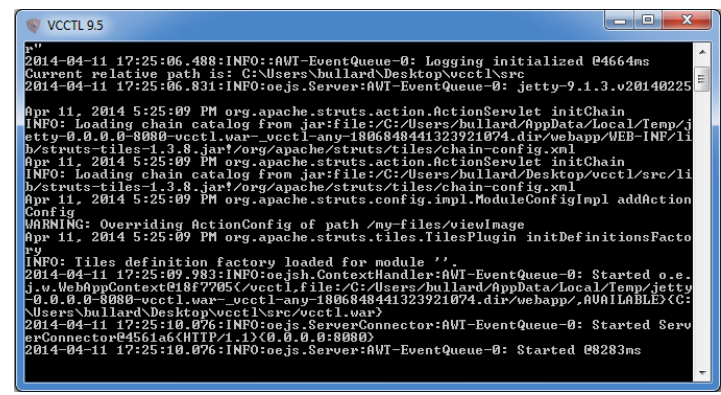

Warning: You must never close the Java console window during an active session of VCCTL. Doing so will cause VCCTL to terminate immediately and, in some cases, a portion of the database that keeps track of your running operations may also become corrupted. It is safest to simply minimize the console window to your desktop tray and leave it there while you are using VCCTL.

A few moments after the Java console window appears, your default web browser should open to the main login page of VCCTL. Again, if your default web browser is Microsoft Internet Explorer, you may observe unexpected or erratic behavior in the software. To avoid this, you can either make some other web browser your default browser, or you can copy the URL for VCCTL's login page into another web browser.

\subsection{Closing VCCTL}

To properly shut down VCCTL, follow these steps:

1. Wait for all running operations to finish. Alternatively, you could cancel all the running operations if needed (this will be described in detail below).

2. Close the web browser

3. Open the Java console window and press $C t r l-C$. After a brief pause, you will be asked, Terminate batch job $(Y / N)$ ?. Type $Y$ and press the Enter button. The Java console window will close. 


\section{Logging On}

VCCTL is a standalone desktop application, but it allows for some data privacy among multiple users on the same computer. For this reason, a session begins with logging on with a user name and password.

The first time you launch a session, you will not have a user name or password, so you must enter yourself in the database by clicking on the New User link. This link will take you to a form shown below:

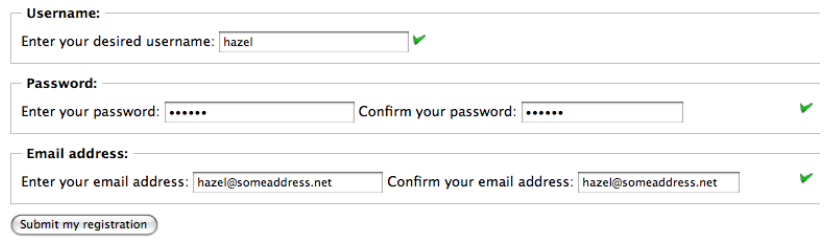

Enter your desired user name in the first box. If the user name is valid and has not been taken by someone else, you will see a green checkmark $(\checkmark)$ as shown. Otherwise, a red $X(X)$ will appear and you will be prompted to choose another name.

Next, enter your desired password. You must confirm the password in the adjacent text field, and if the two text fields agree, you will again see a green checkmark as shown.

Finally, enter your e-mail address in the bottom text box and confirm the address in the adjacent text box. E-mail addresses are not currently used by the application, but may be used in a future release to remotely notify users of completed operations, errors, or other important events. When all the information has been entered properly (i.e., green checkmarks everywhere) then click the Submit button. You will then be taken back to the login page, where you can enter your new user name and password.

\section{The Main Page}

A successful login will open the main page of the application. There are two parts to this page: (1) a section for announcements and streaming video tutorials, (2) a running Menu Bar in gray near the top.

\subsection{Announcements and Tutorials}

This section briefly introduces you to the major features of the VCCTL application and also features a virtual "Lab Tour". The lab tour consists of a series of streaming video tutorials designed to step you through each of the major features and acquaint you with the procedures for running and monitoring different operations. Note that the lab tour requires (1) an internet connection and (2) a QuickTime plugin or other compatible streaming video plugin for your 
browser. Downloading and installing Apple's free QuickTime player should automatically update your browser with the plugin.

The tutorials and this user guide are designed to be used together. One should view the tutorials first, then consult this guide for further details.

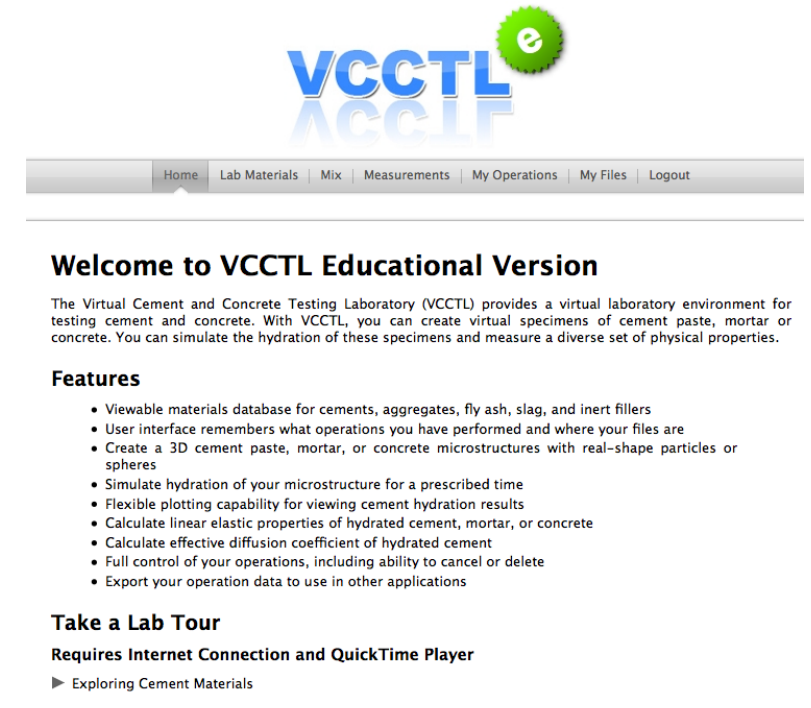

\subsection{The Menu Bar}

The menu bar is a persistent feature on all pages of the VCCTL application, and enables you to quickly navigate from one part of the lab to another. The menu items are

- Home: The main page

- Lab Materials: A multi-part page enabling you to examine the different materials stored in the VCCTL database

- Mix: A two-part page where you can make and hydrate virtual mixes of cement paste, mortar, or concrete

- Measurements: A two-part page enabling you to plot the changes in properties of a material as it hydrates, or to calculate the linear elastic properties, compressive strength, and transport properties of your mix.

- My Operations: Enables you to view the state of your requested operations (queued, running, finished, or cancelled)

- My Files: A listing of all your operations, complete with the ability to view any file in any operation or to export any file for further analysis by another application.

- Logout: Provides a way to logout of the system or switch users. 
Each of these menu items will be described in detail in the following sections.

\section{Lab Materials}

Click on the Lab Materials tab on the main menu, and you will be taken to a page that has a submenu with five tabs as shown below.

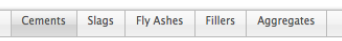

Each tab takes you to a page that enables you to view the properties of the relevant material component in the VCCTL database.

\subsection{Cements}

This page enables you to view the inventory of cements stored in the VCCTL database, along with a number of their physical and chemical properties. There are two sections to this page: (1) View a cement, and (2) View a cement data file. NOTE: Frequently you will see a small question mark icon, $\vec{\theta}$, near a feature on a page. This is a tool-tip icon; clicking on it will open a blue textbox that gives brief information about the feature in question.

\section{View a cement}

The top section of the page has

1. a pull-down menu to select the cement that you want to view,

2. a view of a false-color scanning electron microscope (SEM) image of the cement which has been color-coded to reveal the spatial distribution of the mineral phases,

3. a text box with additional information about the cement, and

4. a collection of quantitative data on the cement, under the heading Cement data that is initially collapsed and hidden when the page opens.

Selecting a different cement in the pull-down menu will update each of these fields to display the relevant data for that cement. The characterization techniques used to generate these data have been described in detail in previous publications $[10,11]$.

\section{Segmented SEM image}

This section shows a single image field from a scanning electron micrograph that has been colored to indicate the locations of the various cementitious mineral phases in the cement powder. A key to the color mappings is provided with each image. 


\section{Information text box}

The text box immediately below the image displays information about the cement, including a brief description, the original source of the cementitious sample, the date when the cement was either received or analyzed, and the fineness measured either by the air permeability test (Blaine, ASTM C204) or by laser diffraction from a dilute suspension of cement powder particles in isopropyl alcohol.

New cements can be uploaded into the database in the form of a single zipped file archive containing a number of specially formatted files. Details on preparing such an archive for upload are provided in Appendix A.

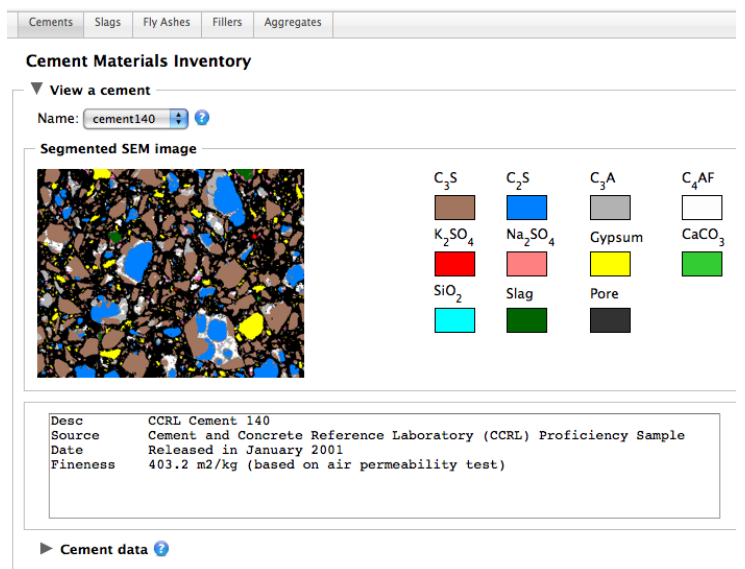

\section{Cement data}

This section is initially collapsed (hidden) when the page opens. It can be displayed by clicking on the small grey triangle next to the Cement data subheading. The fully displayed section contains a number of read-only text boxes and a couple of pull down menus that are described in the following list.

PSD: This is a pull-down menu pointing to the file that contains the information on the particle size distribution (PSD) of the cement. This provides a quick way to alter the PSD of a cement, but it will only take effect if you press the Save or Save As button. An alternative way to modify the PSD of a cement is to change the data in that cement's PSD file. Please refer to the next section, View a cement data file, for more information on how to do this. 


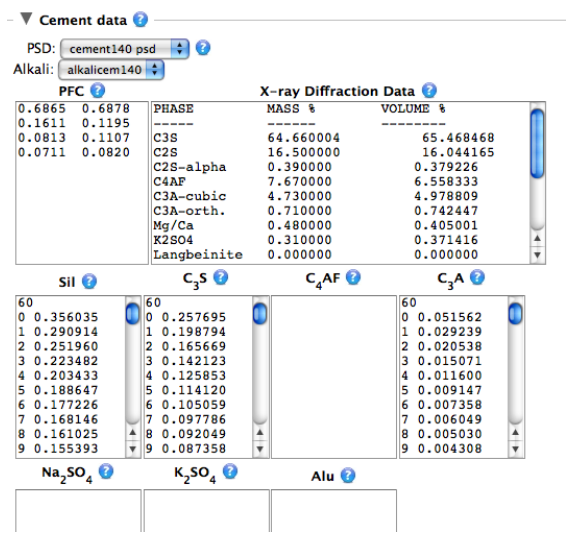

Alkali: This is a pull-down menu pointing to the file that contains the information on the alkali content of the cement, including the total equivalent $\mathrm{K}_{2} \mathrm{O}$ and $\mathrm{Na}_{2} \mathrm{O}$ content as well as the readily-soluble fraction of each. This provides a quick way to alter the cement's alkali characteristics, but it will only take effect if you press the Save or Save As button. An alternative way to modify the alkali characteristics of a cement is to change the data in that cement's alkali file. Please refer to the next section, View a cement data file, for more information on how to do this.

PFC: PFC is an acronym for Phase Fractions in Clinker. The data are displayed as either four or six rows of two columns each. The first row is for alite $\left(C_{3} S\right)^{3}$, the second row for belite $\left(\beta-C_{2} S\right)$, the third row for total tricalcium aluminate $\left(\mathrm{C}_{3} \mathrm{~A}\right)$, and the fourth row for tetracalcium aluminoferrite $\left(\mathrm{C}_{4} \mathrm{AF}\right)$. If a fifth and sixth row are present, they represent arcanite $\left(\mathrm{K}_{2} \mathrm{SO}_{4}\right)$ and thenardite $\left(\mathrm{Na}_{2} \mathrm{SO}_{4}\right)$, respectively. The left column indicates the volume fraction of the associated phase, on a total clinker volume basis, and the right column indicates the surface area fraction of the associated phase, on a total clinker surface area basis. The sum of each column should be 1.0.

X-ray Diffraction Data: This field displays the results of a quantitative X-ray powder diffraction analysis of the cement using Rietveld refinement. The data are not used by the VCCTL software, but are merely present to provide additional information to the user. Changing the data in this box will have no effect on a cement's properties in VCCTL simulations, even if you save the changes.

Sil, $C_{3} S, C_{4} A F, C_{3} A, N_{2} S_{4}, K_{2} S_{4}, A l u$ : These fields display the kernels for the autocorrelation function calculated for the combined silicates, $\mathrm{C}_{3} \mathrm{~S}, \mathrm{C}_{4} \mathrm{AF}$, $\mathrm{C}_{3} \mathrm{~A}, \mathrm{Na}_{2} \mathrm{SO}_{4}, \mathrm{~K}_{2} \mathrm{SO}_{4}$, and combined aluminates, respectively. These data are used to distribute the clinker phases among the cement particles that are created when a mix is requested. One or more of these fields may be blank.

\footnotetext{
${ }^{3}$ Where it is convenient and unlikely to cause confusion, conventional cement chemistry notation is used in this guide: $\mathrm{C}=\mathrm{CaO}, \mathrm{S}=\mathrm{SiO}_{2}, \mathrm{~A}=\mathrm{Al}_{2} \mathrm{O}_{3}, \mathrm{~F}=\mathrm{Fe}_{2} \mathrm{O}_{3}, \mathrm{H}=\mathrm{H}_{2} \mathrm{O}$
} 
Mass fractions of sulfates Three calcium sulfate carriers are recognized: gypsum (calcium sulfate dihydrate), bassanite (hemihydrate), and calcium sulfate anhydrite. This section shows the mass fractions of each of these carriers found in the cement, on a total solids basis. These values may be modified in the database by changing them and then pressing the Save button.

\section{Edit or create a cement data file}

This section is also collapsed when the page first opens. It can be displayed by clicking on the small grey triangle next to the heading View a cement data file.

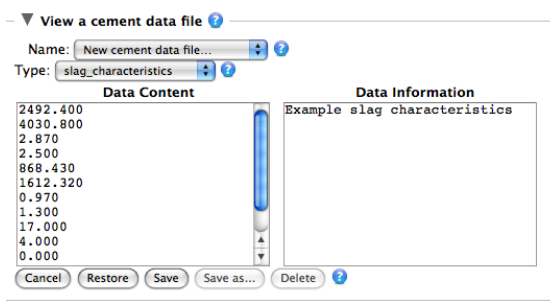

Several auxiliary data files are used by VCCTL to make mixes or simulate the hydration of materials, and this section enables the user to view and modify some of those data files. The left text box contains the actual data, and the right text box contains information on the formatting of the data file.

psd: These files contain information on the particle size distribution of the cement powder. The left column is the equivalent spherical diameter in micrometers (i.e., the diameter of a sphere having the same volume as the irregularly shaped particle), and the right column is the volume fraction of particles having an equivalent spherical diameter between that row and the following row. Therefore, these data describe a discrete probability density function (PDF) for the particle sizes.

alkali characteristics: These files contain information on the potassium and sodium content of the cement on a total solids basis. This data file has four or six rows. The first and second rows are the mass per cent equivalent total $\mathrm{Na}_{2} \mathrm{O}$ and $\mathrm{K}_{2} \mathrm{O}$, respectively. The third and fourth rows are the mass per cent readily soluble $\mathrm{Na}_{2} \mathrm{O}$ and $\mathrm{K}_{2} \mathrm{O}$, respectively. The fifth and sixth rows, if they are present, are the mass per cent $\mathrm{Na}_{2} \mathrm{O}$ and $\mathrm{K}_{2} \mathrm{O}$ added as $\mathrm{NaOH}$ and $\mathrm{KOH}$ to the mix water, respectively.

slag characteristics: There is only one of this kind of data file in VCCTL. It describes the physical and chemical properties of both the slag and the slag gel hydration product. More information on the meaning of each parameter is provided in Section 6.2 on Slags below.

parameters: There is only one of this kind of file in VCCTL as distributed, but users can create others by modifying this one and saving under a different 
name. It catalogs the values of several dozen empirical parameters that are used by the program that simulates hydration of cement pastes. The meaning of each parameter is beyond the scope of this guide. Exercise extreme caution when modifying the parameter file, and only do so if completely familiar with the hydration model. Contact the author for more information about modifying the parameter file.

chemical shrinkage data: Users can calibrate the time scale for hydration simulations using experimentally determined chemical shrinkage data. This file contains the measured data as an example. Users can import other chemical shrinkage data by copying and pasting into the box, preserving the format, and then saving as a new data file.

calorimetry data: Users can calibrate the time scale for hydration simulations using experimentally determined isothermal calorimetry data. This file contains the measured data as an example. Users can import other calorimetry data by copying and pasting into the box, preserving the format, and then saving as a new data file. Users may alter any of the data files and save them by pressing the Save or Save As button below the text boxes. For example, if a user manually modifies the cement 140 psd data file and saves the changes, the modified PSD data will be used any time that cement140 cement is used to create a mix. However, the safer and recommended practice is to save the modified PSD data as a new file called cement140mod psd, and then use the new file to created a modified cement material, thereby preserving all the properties of the original cement140 cement.

\subsection{Slags}

The slag material inventory page consists of a pull-down menu to select the slag to view, along with a textbox displaying the specific gravity of the slag and a second pull-down menu pointing to the file for particle size distribution.

A second section, Slag properties and description, is initially collapsed when the page opens, but can be expanded by clicking on the small grey triangle next to the heading. This section displays the chemical and physical properties that the VCCTL hydration model needs to be able to simulate the hydration of the slag in a blended cement. The properties are self-explanatory, and the tool-tip can be used to gain further information on some of them. Notice that some of the properties relate to the slag itself, some relate to the slag hydration product, and some must be specified for both the slag and the hydration product. 


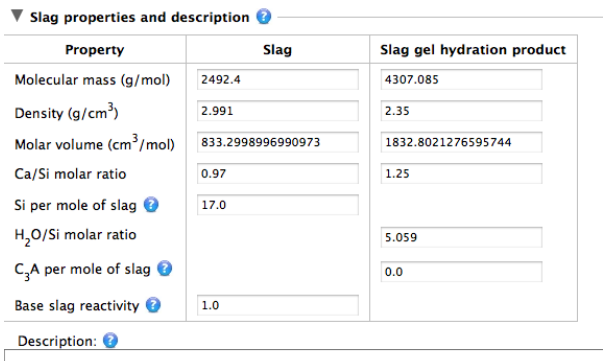

Description: [0

\subsection{Fly Ashes}

The fly ash material inventory page consists of a pull-down menu to select the fly ash to view (there is only one fly ash in the database distributed with VCCTL), along with a textbox displaying the specific gravity of the fly ash and a second pull-down menu pointing to the file for particle size distribution. Unlike slags, fly ash materials consist of multiple phases. Therefore, another field is displayed to indicate whether the phases are to be distributed on a particle basis (i.e., each fly ash particle is a single phase) or on a pixel basis (i.e., each particle contains multiple phases distributed randomly pixel by pixel). The fly ash in the database is set by default to distribute its phases on a particle basis. Clicking the other radio button will cause fly ash phases to be distributed within each particle pixel-by-pixel.

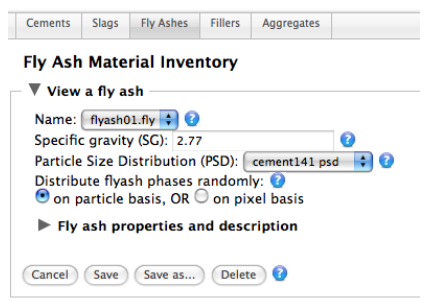

Like the slag inventory page, the fly ash page has a second section, initially collapsed when the page opens, for other fly ash properties. In this case, the fly ash properties are simply the volume fraction of the various phases comprising the fly ash. The seven eligible phases are shown in the accompanying figure. The phase distribution shown is similar to a fly ash that was characterized in 2004 at NIST [14]. 


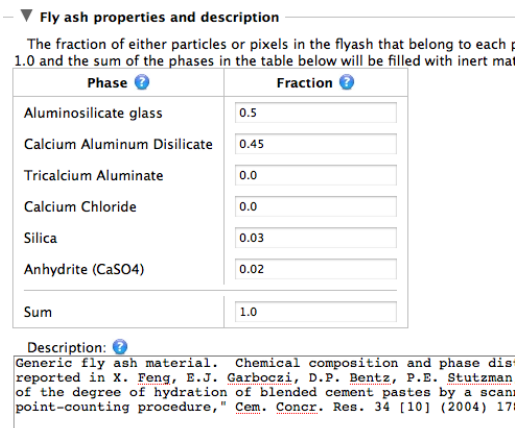

\subsection{Fillers}

Inert fillers have only two properties in the current version of VCCTL: specific gravity and particle size distribution. The inventory of fillers in VCCTL consists of three materials,

1. $\alpha$-quartz $\left(\mathrm{SiO}_{2}\right)$ with a specific gravity of 2.62 ,

2. corundum $\left(\mathrm{Al}_{2} \mathrm{O}_{3}\right)$ with a specific gravity of 4.05 , and

3. periclase $(\mathrm{MgO})$ with a specific gravity of 3.78 .

All three inert fillers use the cement141 psd file by default to describe their particle size distribution.

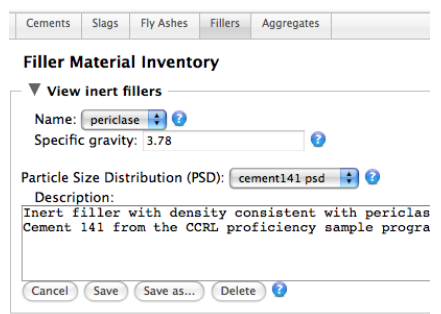

\subsection{Aggregates}

Coarse and fine aggregates are included in the VCCTL database for the purposes of creating mortar and concrete mixes. Three coarse aggregate sources and four fine aggregate sources have been characterized for particle shape, specific gravity, and linear elastic moduli. The shape characterization procedure involves capturing a 3D image of a packing of the aggregate particles, using $X$-ray computed tomography, and then analyzing the shape of individual particles by spherical harmonics [16]. 


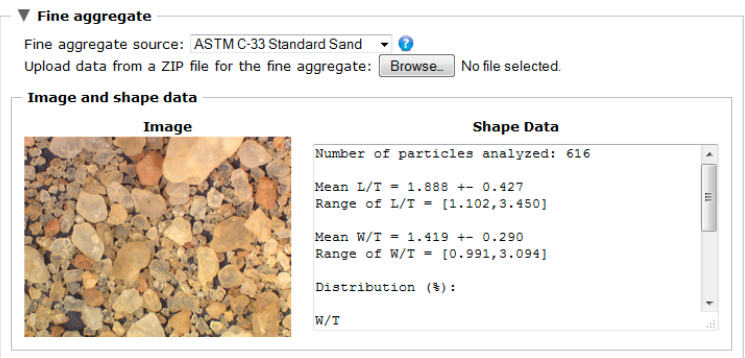

The aggregate materials inventory consists of one section for coarse aggregates and one for fine aggregates. Both sections are collapsed when the page first appears and can be opened by clicking on the small grey triangle next to the respective headings. Each section is identical except for the materials it displays, so the accompanying figures will illustrate the features for the fine aggregates only.

When the coarse or fine aggregate section is expanded, there is a pull-down menu to choose which aggregate source to examine. Below that is a section showing a color photograph of a small sample of the aggregate and an accompanying table that summarizes the gross shape data for the aggregate such as the distribution of ratios of length to width $(\mathrm{L} / \mathrm{W})$ and width to thickness $(\mathrm{W} / \mathrm{T})$. In the table, the mean and range of $\mathrm{L} / \mathrm{T}$ and $\mathrm{W} / \mathrm{T}$ are provided, and below that a table showing the distribution of particles with given $\mathrm{L} / \mathrm{T}$ and $\mathrm{W} / \mathrm{T}$. These data come from the spherical harmonic analysis, but it is useful to remember that the spherical harmonic data in the database contain much more information about particle shape, enough to reproduce the 3D shape of a given particle of any size and rotational orientation in space.

Below the image and shape data, some physical properties of the aggregate are displayed, including the specific gravity, bulk modulus (in GPa) and shear modulus (in GPa). Any of these properties can be changed by the user and saved for the given aggregate (Save button) or saved as a different aggregate source (Save As button). Finally, a text box displays other information about the aggregate, including a descriptive name, the source and intended use of the aggregate and, in some cases, the basic mineralogical content.

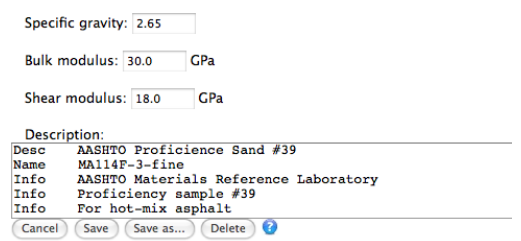

Uploading entirely new aggregate sources, including all the shape data and statistics, can be accomplished using the Upload data from a ZIP file field. As implied by the name, all the properties of the aggregate must be stored in text files with special names, and all the files must be compressed into a single ZIP archive to be uploaded correctly. However, the amount of data and the 
formatting requirements for this feature are extensive, so those interested in using this feature are encouraged to contact the author for instructions on how to prepare the archive for uploading.

\section{$7 \quad$ Mix}

With some knowledge of the types of materials and their properties that are stored in the VCCTL database, we turn now to the part of the software that enables one to create mixes and cure them. This part of the software is accessed through the Mix tab on the main menu bar, and has a submenu for accessing two pages, one for creating a mix and one for simulating its hydration.

\subsection{A Note on the Operating Principles of VCCTL Programs}

An understanding of the underlying operating principles of VCCTL's microstrucure models will clarify the meaning of some of the parameters and options on these pages. VCCTL models are based on a digital-image representation of 3D microstructure. That is, microstructures are mapped onto a regular 3D grid of cubic volume elements, often called pixels or voxels. For cement pastes, each voxel is $1 \mu \mathrm{m}$ on a side, but for larger scale packings of fine or coarse aggregates the voxel size depends on the smallest particle that will be placed. When a cement paste microstructure is created on this voxel grid, each voxel is occupied by exactly one phase (e.g., $\mathrm{C}_{3} \mathrm{~S}$, slag, saturated porosity, etc.). In this way, the programs that lie behind the VCCTL user interface are able to accurately reproduce microstructures that capture the desired phase assemblage, water/cement ratio, particle size and shape distribution, and surface areas.

Simulating hydration of these initial microstructures involves rule-based interactions among the voxels in the microstructure. The rules are designed to mimic the various chemical reactions and mass transport that occur during hydration. As a result, the 3D digital image microstructure is incrementally changed to simulate microstructure development. This process introduces new hydration product phases such as $\mathrm{C}-\mathrm{S}-\mathrm{H}$ gel, $\mathrm{CH}$, ettringite, and several others.

The full details of the operating principles for the digital image approach can be found in other publications [1,2]. A discussion of computational tools that have been developed to analyze the properties of digital image microstructures can be found in Ref. [18]. Some of the artifacts and limitations of digital image approaches are described in Ref. [17].

\subsection{Prepare Mix}

This page is used to create an initial 3D cement microstructure consisting of particles of cement clinker, calcium sulfate carriers, and other selected compounds in water, as well as simulated packings of fine and coarse aggregates 
when the paste is acting as the binder in a mortar or concrete. There are three main steps involved in creating the mix:

1. specifying the solid cementitious binder properties,

2. specifying the mix proportions of the solid portion of the binder with water and aggregate, and

3. specifying the system size and other miscellaneous simulation parameters.

\section{Specifying the solid cementitious binder}

The principal component of the binder is one of the portland cements, which may be selected from among those in the database using the pull-down menu selector. Once the cement is selected, the volume fractions and surface area fractions of the clinker minerals may be modified for the mix if desired by expanding the section Modify phase distribution of the clinker. The default values displayed in the tables are those stored in the database for the corresponding cement when it was characterized as already described. The table keeps a running sum of the two columns, which should both add to 1.0. If the user modifies the values in the columns in such a way that one or both columns do not sum to 1.0, the columns will be re-normalized to 1.0 when the mix is created.

The chosen cement also has default values for the mass fractions of the calcium sulfate carriers (dihydrate, hemihydrate, and anhydrite) which can be reviewed and modified by expanding the section headed Modify calcium sulfate amounts in the cement. Remember that the displayed values are given on a total solid cement basis, and that all values are displayed as fractions, not percentages.

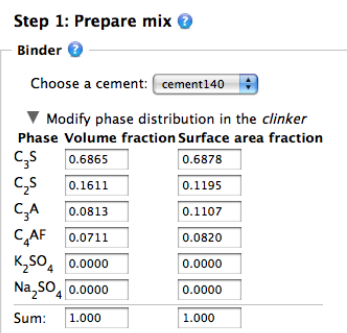

The last part of specifying the solid binder is to select the type and quantity of any supplementary cementitious materials (SCMs) or fillers. The categories for these materials are (1) fly ash, (2) slag, (3) inert filler, (4) silica fume, (4) limestone $\left(\mathrm{CaCO}_{3}\right)$, and (5) free lime $(\mathrm{CaO})$. For fly ash, slag, and inert filler, the materials may be selected from the accompanying pull-down menu selector from those available in the database, as already described in Section 6. For silica fume, limestone, and free lime, the composition and specific gravity are 
assumed fixed, and the user is asked only to specify the particle size distribution by selecting the file for the PSD from one of those in the database. Recall that the PSDs associated with each file can be viewed on the Lab Materials page as described in Section 6.1. Once the material and characteristics are selected, the user may specify either the mass fraction or volume fraction that the material will occupy on a total solid binder basis. This is equivalent to specifying a replacement level for each component. Note that the total fraction of cement in the binder is reduced as the fractions of these other materials is increased. If the mass fraction is specified, then the volume fraction will be automatically calculated and displayed based on the specific gravities of all the components. Similarly, specifying the volume fraction will cause the mass fraction to be automatically calculated and displayed.

\section{Specifying the mix proportions}

When the solid portion of the binder is specified, the next section can be used to specify the water/binder mass ratio $(\mathrm{w} / \mathrm{b})$ using the pull-down menu selector. The options for the $\mathrm{w} / \mathrm{b}$ value cover the range that is normally used for concrete and also include the value 0.485 , the latter of which is used for ASTM C109 mortar cube compressive strength specimens. Text fields are also available for viewing the mass fractions and volume fractions of solid binder and water correponding to the selected $\mathrm{w} / \mathrm{b}$ ratio. Modifying either a volume fraction or a mass fraction will automatically update the other fields.

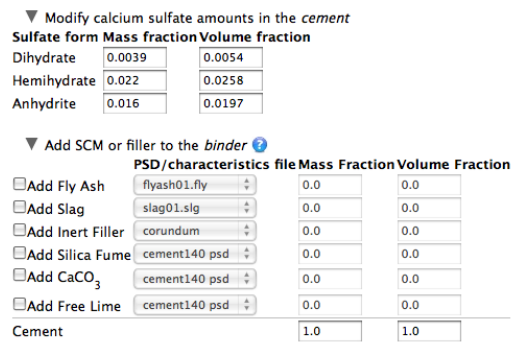

This section also includes the option to add coarse or fine aggregate to the mix to create a mortar or concrete. To add aggregate, click on the checkbox next to the coarse or fine aggregate. When this is done, the text boxes for the mass fraction and volume fraction, initially disabled, are enabled and the user can type in the desired mass fraction or volume fraction, on a total mortar or concrete basis.

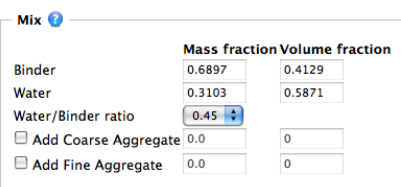

In addition, when one of the aggregate checkboxes is selected, a new section appears below it, intially collapsed, called Change properties. Expanding this 
section enables the user to specify the aggregate source, specific gravity, and grading (or size distribution).

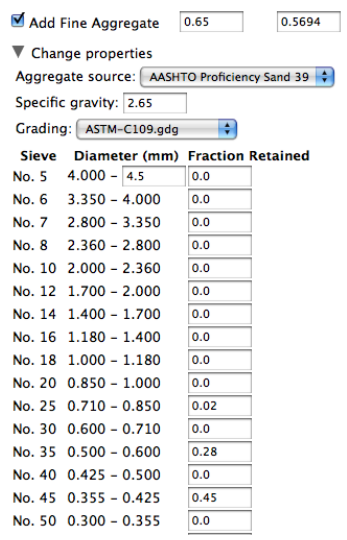

Notice that changing the specific gravity of the aggregate causes the volume fractions of the aggregate, binder, and water all to change in response. Finally, aggregate grading is displayed in tabular form using the customary sieve designations to indicate the size bins that are used. The user may specify the grading by typing in the mass fraction of aggregate retained in each sieve. The sum of the mass fractions in the table should be 1.0, but it will be normalized to 1.0 if not. VCCTL comes with one pre-selected fine aggregate grading that corresponds to the grading specified in the ASTM C 109 mortar cube compressive strength test. The coarse aggregate section also comes with a default aggregate grading, but the default grading does not correspond to any particular test.

Note: The aggregate grading specified manually by the user can be saved in the database for easy retrieval in subsequent mixes.

\section{Specifying simulation parameters}

After the mix proportions are specified, the user may optionally select or modify any of several simulation parameters.

Random number generator seed: When the virtual microstructures are created, random numbers are used to randomly select the positions of particles as they are added, to stochastically distribute the clinker phases within the constraints of the two-point correlation functions, and a variety of other tasks. The random number generator used in the VCCTL programs require an initial value, called a "seed" which must be a negative integer. Under ordinary circumstances, the seed value will be chosen automatically, but the user may also specify a particular seed value, if desired, by checking the box and typing any integer other than zero in the text box; a value of zero will cause VCCTL to automatically generate a non-zero pseudo-random integer as the seed. 


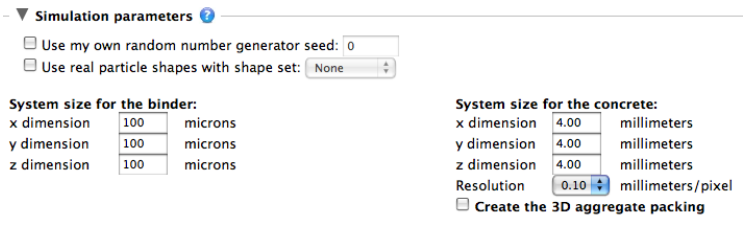

Real particle shapes: Check this box if you want the cement particles in the microstructure to have realistic shapes. As already described, the 3D shapes of aggregate particles have been characterized by spherical harmonic analysis and stored in the VCCTL database to recreate realistic aggregate packings. A nearly identical procedure has been used to characterize the particle shapes of several different portland cement powders, except that X-ray microtomography is required to obtain sufficient spatial resolution to characterize the particles using spherical harmonics [20]. Realistic cement particle shapes will be placed in the microstructure by marking the check box and selecting the shape set from the pull-down menu selector. Note that requesting realistic particle shapes will require significantly more time to create the microstructure, sometimes two or three times as long. If the real particle shape checkbox is not selected, all the cement particles will all be digitized spheres. Even if real particle shapes are requested, fly ash particles will remain spherical to be consistent with their usual morphology.

Flocculation: Choosing this option will cause the particles placed in the box to be displaced in random directions, locking with any other particles with which they come in contact during the process. The degree of flocculation is the fraction of particles that you wish to have connected into a single agglomerate. A value of 0.0 will cause no flocculation, whereas a value of 1.0 will ensure that every particle in the system is attached to a single agglomerated structure. Intermediate values between 0 and 1 will cause a number of disconnected agglomerates to form, with the number of agglomerates decreasing, and their average size increasing, as the degree of flocculation approaches 1.0.

Dispersion: Checking this box will ensure that every particle is separated from every other particle in the system by a distance of one or two voxels, thereby simulating the influence of a strong dispersing agent.

System size for the binder: The number of voxels in each linear dimension of the 3D grid can be chosen by the user, the default value being 100 in each direction. As already mentioned, each cubic voxel in the cement paste is 1 ?m on a side, so the number of voxels in each dimension is numerically equal to the linear dimension. Note that larger values of the linear dimensions will require significantly more time to create the microstructure; the time required to create a microstructure scales linearly with the number of voxels $\left(N_{\mathrm{cp}}\right)$ in the grid, which can be calculated according to the equation

$$
N_{\mathrm{cp}}=N_{x} N_{y} N_{z}
$$


where $N_{x}, N_{y}$, and $N_{z}$ are the numbers of voxels in the cement paste in the $x$, $y$, and $z$ directions of the grid.

System size for the concrete: This section appears only if fine or coarse aggregate is being added to create a mortar or concrete. The default values for the linear dimensions and resolution are determined by the grading specified by the user. However, these values may be changed subject to the following constraints:

- The minimum linear dimension of the concrete system must be at least twice as big as the opening of the coarsest sieve that retains any aggregate.

- The resolution chosen from the pull-down menu selector must be no more than half the opening of the finest sieve that retains any aggregate.

These constraints are used to ensure that each aggregate particle in the grading fits into the box and can be approximately visualized in the packing. If either of these constraints is violated, an alert box will appear with a message describing which constraint was violated. Clicking the $O K$ button on the dialog box dismisses it and also causes the offending dimension or resolution to be reset to an acceptable value that satisfies the constraints.

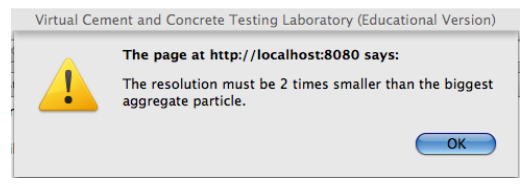

Note: Even when coarse or fine aggregates are included in the mix, the 3D aggregate packing is not created by default. To force the creation of an aggregate packing, check the next to Create the 3D aggregate packing.

Note: Creating a virtual aggregate packing often requires significant computational resources because the shapes are created individually from the spherical harmonic coefficients stored in the database. As with the cement paste system, the time required to create a $3 \mathrm{D}$ aggregate packing will scale linearly with the number of voxels $\left(N_{\mathrm{ap}}\right)$ in the aggregate packing, which can be calculated according to the equation

$$
N_{\text {ap }}=\frac{L_{x} L_{y} L_{z}}{\lambda^{3}}
$$

where $L_{x}, L_{y}$, and $L_{z}$ are the linear dimensions of the grid in $\mathrm{mm}$, and $\lambda$ is the resolution in $\mathrm{mm}$ /voxel.

Note: If fine or coarse aggregates are included in the mix, then a "virtual" boundary plane, parallel to the $y z$-plane, is inserted in the middle of the microstructure, regardless of whether or not an aggregate packing is requested. The plane cannot be seen, but cement particles are forbidden to intersect it, a condition that simulates interfacial transition zone (ITZ) in mortar and concrete arising from the packing limitations of cement particles near an aggregate surface. The properties of the ITZ are important in determining the mechanical 
properties and transport properties of mortar and concrete [6], so it is important that the ITZ be recreated in the virtual cement paste microstructure so that its properties can be subsequently computed and incorporated into calculations of mortar and concrete properties.

\section{Saving the mix}

The last steps in creating a mix are to give the mix a unique name (i.e. one that does not already exist in the database) and execute the mix creation program. Type a name for the mix in the text box provided. Names can include upper case or lower case letters, numbers, or spaces. After typing the proposed name and clicking anywhere outside the text box, the software will validate the name. A green checkmark $(\checkmark)$ next to the name indicates that it is valid.

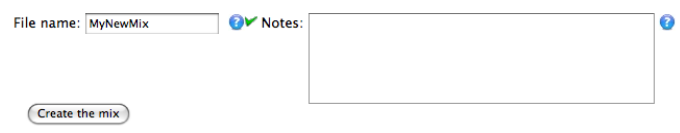

A red $X(X)$ indicates that the name has already been used for an existing mix.

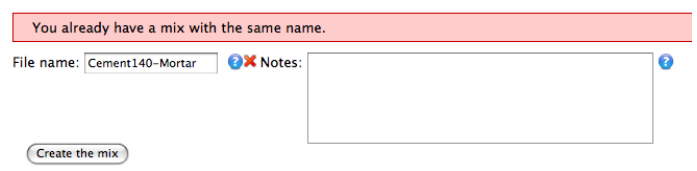

Once a valid name has been chosen, click on the Create the mix button to begin creating the mix. The status of the mix operation can be checked on the My Operations page, as described in detail in Section 9.

\subsection{Hydrate Mix}

The NIST cement hydration model $[1,2]$ operates directly on 3D digital images of cement particle microstructures created as described earlier. Execution of the model requires a starting microstructure, information about the curing conditions (thermal conditions and moisture state), and the frequency at which to output various data for later analysis.

To begin hydrating a mix, first select the mix name from the pull-down menu selector. This is the name chosen when the mix was created. You may also specify the apparent activation energies of the three major net hydration processes that can occur, which are (1) cement hydration, (2) pozzolanic reactions, including fly ash hydration and silica fume reactions, and (3) slag hydration. The default values that are provided reflect sensible values based on a survey of the literature (for example, see ASTM C 1074). The section for modifying the activation energies is collapsed when the page first opens, but may be expanded by clicking on the small grey triangle next to the heading Reaction activation energies. 


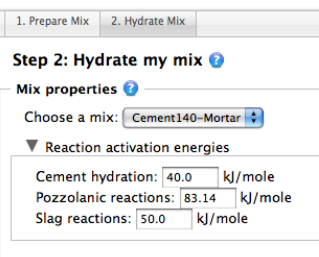

\section{Thermal conditions}

The thermal condition of the hydrating material may be (1) isothermal, (2) semiadiabatic, or (3) adiabatic.

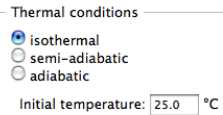

Isothermal: The material is in diathermal contact with a constant-temperature reservoir and heat transfer is sufficiently rapid to maintain the material at the temperature of the reservoir. The default value is $25^{\circ} \mathrm{C}$.

Semi-adiabatic: Heat generated by hydration reactions is transferred to a surrounding reservoir that is at a fixed temperature, but the transfer rate is slow enough that some of the generated heat causes the material temperature to increase. If this option is selected, then not only the initial temperature of the material must be specified (default $25^{\circ} \mathrm{C}$ ), but also the temperature of the surrounding reservoir (default $25^{\circ} \mathrm{C}$ ) and the effective heat transfer coefficient, in units of $\mathrm{W} / \mathrm{K}$ (default 0.0 , which is the adiabatic condition).

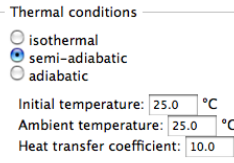

If the material being hydrated is a mortar or concrete, a section will be present to specify the aggregate's initial temperature and its heat transfer coefficient with the binder. This feature can be useful to simulate the influence of chilled aggregate on a hot day. However, the feature has no effect if isothermal conditions are chosen.

Adiabatic: The material is perfectly insulated from its environment, so all the heat generated by hydration reactions is used to increase the temperature of the material. If this option is chosen, only the initial temperature of the material must be specified. The default value is again $25^{\circ} \mathrm{C}$. 


\section{Hydration time}

This section is used to specify the total length of time that the material should be hydrated. The time is specified by entering the desired number of days in the text box provided or, alternatively, by specifying a maximum degree of hydration at which the simulation will terminate.

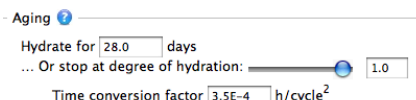

The other required element is the time conversion factor. The NIST hydration model proceeds in repeated cycles, each of which has a dissolution step, a diffusion step to transport dissolved components through the solution, and a reaction step. These cycles have no intrinsic time scale, so one must define an assumed relationship between time (in hours) and computational cycles. In the current version of VCCTL, hydration time $(t)$ in hours is related to the number of computational cycles $(n)$, according to the parabolic relation

$$
t=\beta n^{2}
$$

where $\beta$ is the time conversion factor that must be specified, in units of $h / \mathrm{cycle}^{2}$. The default value of $3.5 \times 10^{-4}$ works reasonably well for a number of cements but is not optimized to any particular cement. To optimize the time conversion factor, a calibration of the simulation against some experimental measure of the degree of hydration, chemical shrinkage, or isothermal heat release should be made.

Note: The parabolic relation employed for the time scale is not used for any fundamental reason, but only because it often provides a reasonable fit to experimental measurements of the progress of hydration with time, provided that the time conversion factor has been calibrated adequately.

Note: The choice of time conversion factor has no influence on the calculations other than to dilate or contract the time scale for the simulation.

\section{Using experimental data to simulate kinetics}

Cement hydration kinetics at early times can be calibrated directly using experimental data as an alternative to the time conversion factor. If the cement paste has been characterized by continuous chemical shrinkage measurements at any isothermal temperature at or near the desired curing temperature ${ }^{4}$, the user can upload the relevant experimental data in the appropriate format on the Lab Materials page, check the appropriate radio button in this section, and select the appropriate data file from the pull-down menu. See Section 6.1 for further details about importing experimental data.

\footnotetext{
${ }^{4}$ The temperature at which the experimental data were obtained must be isothermal and should be within $\pm 20^{\circ} \mathrm{C}$ of the desired curing temperature, or else VCCTL's method of using an Arrhenius correction to the temperature may become less accurate.
} 
If one of these options is chosen, the VCCTL model will track the simulated progress of hydration in terms of the simulated chemical shrinkage or the heat signature, and will make comparison to the available experimental data to determine elapsed time for any degree of hydration. If the simulation reaches a time beyond which the experimental data terminate, the models will attempt a quadratic extrapolation to later times.

\section{Saturation conditions}

The two moisture condition options are saturated or sealed. The saturated condition means that the cement paste is in contact with a reservoir of excess water. As free water in the capillary pores is consumed by the hydration reactions, it is immediately replaced by water from the reservoir. Note, however, that once the capillary porosity reaches its percolation threshold and becomes disconnected, water replacement is no longer possible even under saturated conditions. The sealed condition means that free water consumed during hydration is not replaced. Instead, the capillary pores are progressively emptied of water, starting in those pore regions with the largest effective diameters.

Saturation conditions

$\odot$ saturated
sealed

\section{Simulation parameters}

This section is collapsed when the page first opens because it will not usually be of interest. It can be expanded by clicking on the small grey triangle next to the heading. The only simulation parameter that can be modified in VCCTL is the value of the seed for the random number generator.

\section{Data output}

This section is collapsed when the page first opens. It can be expanded by clicking on the small grey triangle next to the heading. This section can be used to specify how frequently (in hours of simulated age) the hydration model will output certain properties to data files.

Evaluate percolation of porosity: Percolation properties like this one tend to consume significant computational time to calculate because the calculations involve nested iterations over most or all of the system voxels. Therefore, it is recommended that percolation properties not be evaluated any more often than necessary. The percolation state of the capillary porosity determines the point at which water can no longer be absorbed from an external source to maintain saturated conditions. Once the hydration model detects that the pore space is depercolated, this calculation will no longer be made no matter what value is typed into the text box. The default value is $1.0 \mathrm{~h}$. 
Evaluate percolation of total solids: This property provides an indication of initial setting time, so it should be evaluated fairly frequently to obtain a reasonable assessment of setting time of a material. The default value is $0.5 \mathrm{~h}$.

Output hydrating microstructure: The state of the entire 3D microstructure can be saved at regular intervals during the hydration process. Subsequent calculations of the linear elastic moduli, compressive strength, and transport properties of the material at a particular state of hydration can only be made if the microstructure is available at that state. The default value is $72.0 \mathrm{~h}$.

Output hydration movie: If this box is checked, the model will output the microstructural state of a $2 \mathrm{D}$ slice at time intervals specified by the user. These data are then used to create a movie of the progress of hydration that can be viewed on the My Files page. Note that the default time interval of $0.0 \mathrm{~h}$ will result in no movie being created. See Section 10.2 for more information about the available options when viewing images and movies.

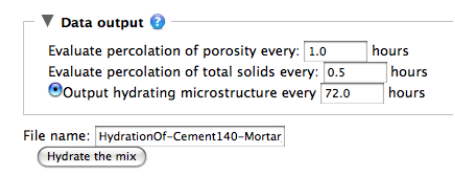

\section{Executing the hydration simulation}

To execute the simulated hydration operation, provide a unique name for the operation in the box next to File name at the bottom of the page. The default name is the name of the mix with the prefix HydrationOf- prepended. The default name can be modified, but must be unique. The software validates the name before and will indicate a green $\checkmark$ (okay) or a red $\mathcal{X}$ (invalid) next to the name. Once a valid name is selected, click on the Hydrate the mix button to launch the operation. The status of the hydration operation can be checked on the My Operations page, as described in detail in Section 9.

Note: One or more hydration operations for a particular mix can be requested and launched even before the mix operation has been completed. If the mix operation is still running when the hydration request is made, a message will appear saying, The mix you want to hydrate is not ready now. The hydration will begin as soon as the mix preparation is finished. In other words, the hydration operation is queued to run as soon as the mix operation completes and a starting microstructure is available.

\section{Measurements}

In VCCTL, 63 properties are continuously tracked during a hydration operation, and all of them are stored in a data file that can be accessed for online plotting or exporting to a spreadsheet for further analysis. See Appendix $C$ for 
a listing of all the available hydrated properties. In addition, the linear elastic moduli, compressive strength and transport properties of cement pastes, mortars, or concretes can be calculated on hydrated microstructures. The Measurements page provides access to all these virtual measurement capabilities.

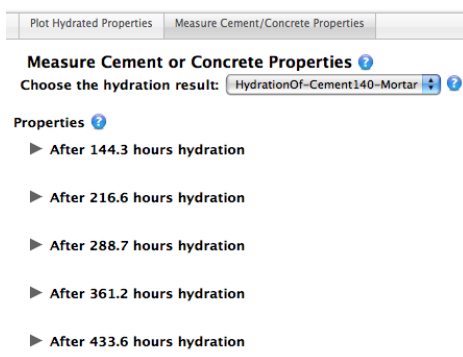

Clicking on the Measurements tab of the main menu bar opens a page with a submenu having two options: (1) Plot Hydrated Properties, and (2) Measure Cement/Concrete Properties.

\subsection{Plot Hydrated Properties}

This page provides a flexible inline plotting capability for plotting the evolving properties of a hydrating material. To create a plot, first select the desired property to use for the $x$-axis from the pull-down menu selector. The default $x$-axis is Time, in hours, but any other hydrated property can be selected as well.

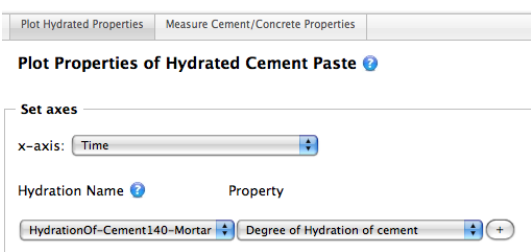

With the $x$-axis selected, any number of properties can be simultaneously plotted on the $y$-axis, including multiple properties from a single hydration simulation, or a comparison of the same properties for different hydration simulations. To create a plot, first select a hydration operation on the left pull-down menu selector (under the heading Hydration Name), and then select the desired property to plot in the right pull-down menu selector (under the heading Property). Doing so will immediately create the plot in the Plot result area. 


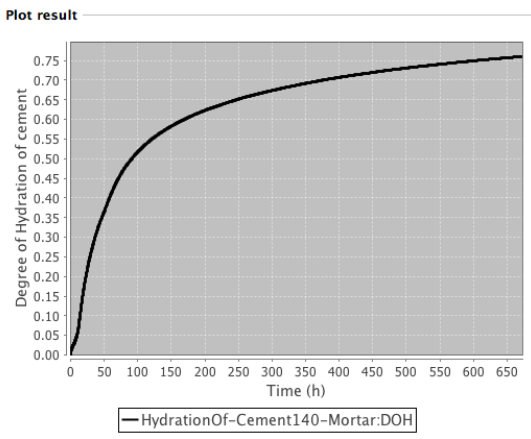

The plot is accompanied by a legend indicating both the hydration operation and the property, separated by a colon.

Click the " + " button next to the first property in the Set axes section to plot a second property on the same plot. This will create a second row with the same pull-down menus repeated.

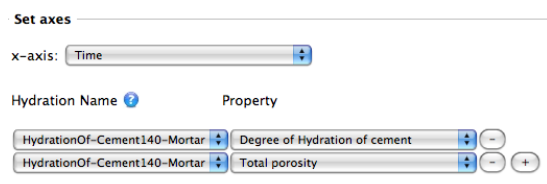

The example shown below plots both the degree of hydration and total capillary porosity against time for the same hydration operation. Notice that the legend is updated to display the information for each curve in the plot.

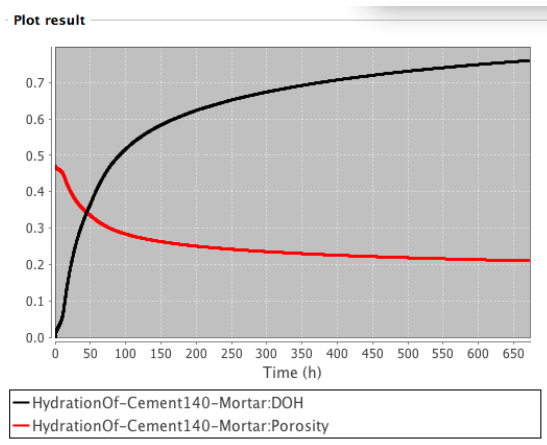

Note: When plotting multiple properties on the same graph, the properties must all have commensurate units. For example, both the degree of hydration and the total porosity are dimensionless quantities, so they can be plotted together. However, the degree of hydration and isothermal heat release have different units, so they cannot be plotted simultaneously. The pull-down menu selectors automatically exclude incommensurate properties.

You can also compare properties from different hydration operations to analyze differences in the behavior of the two systems. The following example adds to the example just given by plotting the degree of hydration 
for another hydration operation. Notice that the second hydration operation, HydrationOf-ASTMC109-02, only ran for $24 \mathrm{~h}$.

Plotted curves can be removed from the graph by clicking on the "-" button next to the corresponding row in the Set axes section.

Note: Under some conditions, the generated plot may not automatically rescale its axes in an optimal way. This can be corrected by simply clicking once anywhere within the region of the plot itself.

It is also possible to zoom in on a narrow range of the $x$-axis. To do so, place the mouse within the plot field at the beginning $x$ value. Hold the mouse button down and drag to the ending $x$ value. Releasing the mouse will automatically generate the rescaled plot. Again, to go back to the optimal scaling needed to show all the data, just click once within the plot region.

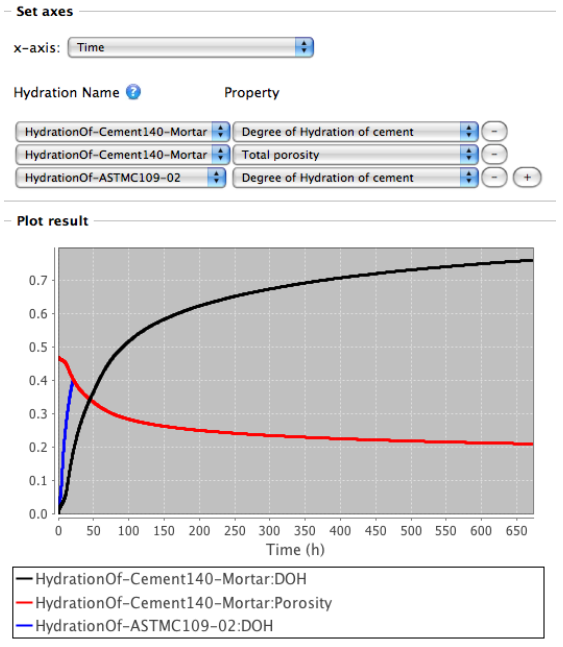

Finally, a high-resolution plot can be created in separate pop-up window by clicking on the High resolution version link below the legend. The same zooming features apply to the high-resolution plot as for the regular plot.

Note: When a hydration operation is running, its progress can be monitored by going to this plotting page. The default plot, which has time on the $x$-axis, will show how much simulation time has been reached, even if the operation has not completed.

\subsection{Measure Cement/Concrete Properties}

Clicking on this subtab opens a page for calculating the linear elastic properties, compressive strength, and the effective diffusivity of cement pastes, mortars, or concretes that have been hydrated. The desired hydration operation can be chosen from the pull-down menu selector. When this is done, a listing of the available hydration times for this operation appears under the heading Properties. 
Recall that, when a hydration operation is requested, you have the option to select the time intervals at which to output the partially hydrated 3D microstructures. For example, if you chose $72.0 \mathrm{~h}$ for this interval, microstructures were saved at approximately $72 \mathrm{~h}, 144 \mathrm{~h}, 216 \mathrm{~h}$, etc. These are the same times that now appear in the list on this page, as individually collapsed sections, any of which can be expanded by clicking on the small grey triangles next to the headings.

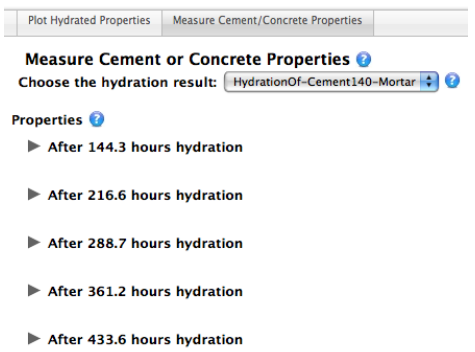

When one of these individual sections is expanded, it reveals two buttons, one to calculate the elastic properties (effective linear elastic moduli) and one to calculate the transport properties (relative diffusivity and formation factor). Clicking on these buttons launches the finite element models that calculate these properties on the digital image [15].

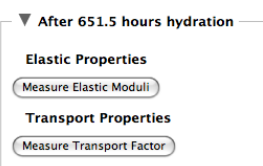

The finite element calculations take significant computational time to complete, about $20 \mathrm{~min}$ to $30 \mathrm{~min}$ for a typical $100 \times 100 \times 100$ system. Once the calculations begin, the relevant section of this page will display several boxes that will eventually contain the results of the calculations. While the calculations are ongoing, however, these boxes will display the message Measuring .... The data that are displayed in these boxes when the calculations are completed is described now. 


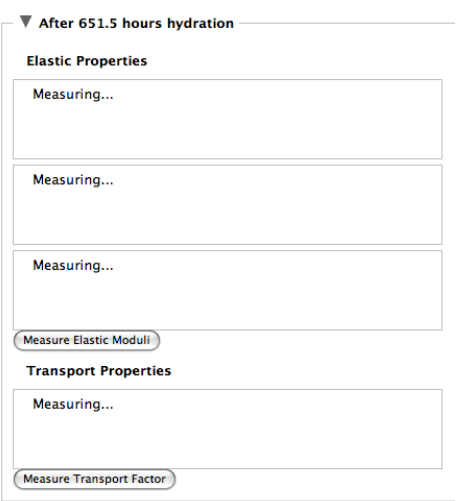

\section{Elastic properties results}

There are three text boxes to display the elastic properties results:

- The first box provides a summary of the effective elastic properties of the cement paste, including the bulk modulus, $K$ in GPa, shear modulus, $G$ in GPa, Young's modulus, $E$ in GPa, and Poisson ratio, $v$. If the material is a mortar or concrete, this box will also display the effective elastic properties of the mortar or concrete. These latter properties are calculated with Differential Effective Medium Theory (D-EMT) [19], and include the effective $K, G, E$, and $v$. The D-EMT model takes the cement paste elastic properties, aggregate elastic properties, and the properties of the ITZ (also gleaned from the finite element calculations on the cement paste near the aggregate surface, as described below), as well as the volume fractions of the paste, aggregates, and air voids. In addition to these properties, a mortar or concrete will also have the estimated compressive strength displayed in the box, in $\mathrm{MPa}$, which is estimated from the empirical relationship between Young's Modulus and compressive strength described by Neville [21].

- The second box shows the relative contribution to the elastic properties of each phase in the microstructure. For each phase in turn, the following data are provided

1. the volume fraction of the phase

2. the mean value, over all the voxels of the given phase, of the bulk modulus calculated using the linear elastic relation between the local stress and the macroscopically applied strain

3. the fraction that this value is of the total effective bulk modulus of the material

4. the mean value (defined as in (2)) of the shear modulus

5. the fraction that this value of shear modulus is of the total effective shear modulus 
6. the mean value (defined as in (2)) of Young's modulus

7. the fraction that this value of Young's modulus is of the total effective Young's modulus

- The third box contains information about the variation of the elastic properties of the cement paste as a function of distance perpendicular to the aggregate surface. The data appear in five columns, identified as

1. distance from the aggregate surface $(\mu \mathrm{m})$

2. $K$ for the paste $(\mathrm{GPa})$

3. $G$ for the paste $(\mathrm{GPa})$

4. E for the paste (GPa)

5. $v$ for the paste.

These data are calculated directly from the finite element model results and are used to determine the elastic properties of the ITZ. The ITZ properties are determined by averaging these data over thickness of the ITZ, which is approximately equal to the median particle size of the starting cement powder [6].

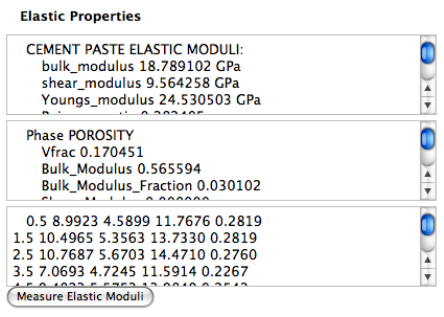

\section{Transport properties results}

Calculated transport properties are displayed in one box, and consist of the relative diffusion coefficient in the $x, y$, and $z$ directions. Here the relative diffusion coefficient is defined as the effective diffusion coefficient of mobile solution species in the material, divided by the diffusion coefficient of the same species in pure pore solution having the same composition. In addition, the average relative diffusion coefficient over all three dimensions and the formation factor are displayed in the text box. The formation factor is the inverse of the average relative diffusion coefficient as defined here.

Following the value of the formation factor, the text box displays phasespecific information similar to that displayed for elastic properties above. For each phase in turn, the following data are provided:

- the volume fraction of the phase

- the relative conductivity of the phase, that is, the conductivity of mobile species through the phase itself divided by the conductivity of the same species through pure pore solution of the same composition; saturated 
porosity has a relative conductivity of 1.0 by definition, and solid dense phases have a relative conductivity of 0.0

- the fraction that this phase contributes to the overall effective conductivity of the composite materials in each orthogonal direction.

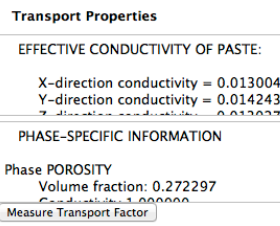

\section{My Operations}

The My Operations page is the central location for checking the status of a user's operations. This guide has already discussed all of the major operations that can be executed in VCCTL, but now is a good time to list all the types in summary.

- Microstructure: Creation of a cement paste microstructure

- Aggregate: Creation of a mortar or concrete aggregate packing

- Hydration: Simulating the hydration of a cement paste

- Elastic-moduli: Calculation of elastic moduli of a cement paste, mortar, or concrete

- Transport-factor: Calculation of relative diffusion coefficients and formation factor

On the My Operations page, each operation for a user is listed in one of four sections, depending on their status: queued, running, finished, or cancelled.

\subsection{Queued Operations}

When an operation is requested on one of the VCCTL pages, it is given an entry in the database and is placed in a queue waiting to be run. Some operations will leave the queue and begin running almost immediately, but some others, such as hydration operations waiting for their microstructure to be created, will remain in the queue for some time. While in the queue, a new directory is created to store the results, and an input file is created for the $C$ program that will process the input and generate the results. On the My Operations page, operations in the queue appear in a yellow table at the top of the page, with information about the type of operation (see the previously enumerated list for types) and the time of the request displayed. 


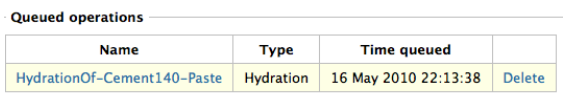

At the right of the entry is a link to delete the operation from the queue. Clicking the Delete link will remove the operation request from the database and delete the directory associated with that operation.

\subsection{Running Operations}

While an operation is running, it appears in a green table in this section. The name of the operation appears as a link. If the name is clicked, the My Files page is opened to the operation in question so the user can examine the files that have been generated for the operation. See Section 10 for more detail on the My Files page. In addition to the name, the operation type and the time started are displayed.

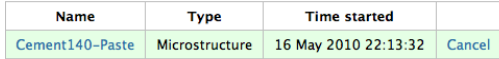

At the right of the entry is a link to cancel the operation. Clicking the Cancel link will terminate the $\mathrm{C}$ program that is running the operation, and the operation will be moved to the Cancelled operations section of the page. The input and output files associated with the operation will remain intact, and any data generated up to the point of cancellation can be viewed on the My Files page.

\subsection{Finished Operations}

When the $C$ program running an operation has finished (either successfully or unsuccessfully), the operation is moved from the Running operations section to this section. It appears as an entry in a blue table. As before, the name of the operation appears as a link that redirects the page to the operation's entry in the My Files page. In addition to the name, the operation type is displayed, as well as the time the operation started and finished.

\begin{tabular}{|l|l|l|l|l|}
\hline \multicolumn{1}{|c|}{ Name } & \multicolumn{1}{|c|}{ Type } & \multicolumn{1}{|c|}{ Time started } & \multicolumn{1}{|c|}{ Time finished } & \\
\hline HydrationOf-Cement152- & Transport- & 16 May 2010 & 16 May 2010 & Delete \\
Mortar/Transport-factor-9 & factor & $20: 08: 12$ & $20: 11: 05$ & \\
\hline $\begin{array}{l}\text { HydrationOf-Cement152- } \\
\text { Mortar/Elastic-moduli-9 }\end{array}$ & Elastic-moduli & $\begin{array}{l}16 \text { May } 2010 \\
20: 08: 07\end{array}$ & $\begin{array}{l}16 \text { May } 2010 \\
20: 43: 34\end{array}$ & Delete \\
\hline
\end{tabular}

At the right of each entry is a link to delete the finished operation. Clicking the Delete link will delete all input and output files generated by the operation and will remove the operation's listing from the database.

Note: The deletion of an operation cannot be undone, so use this feature with caution. 


\subsection{Cancelled Operations}

If a running operation is cancelled, its entry appears in this pink table. In VCCTL, the operation cannot be restarted, but it can be deleted. Clicking on the Delete link at the right of the entry will remove the operation's listing from the database and will also remove all input and output files associated with the operation from the computer. The deletion of a cancelled operation cannot be undone.

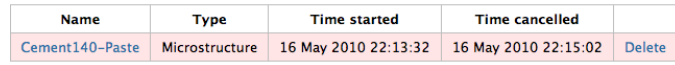

\section{My Files}

Other than the Measurements page, the My Files page is the main location for viewing the progress and results of an operation. The input and output files associated with an operation can be viewed here or optionally exported to the computer's Desktop for further examination.

The My Files page has five sections, one for each type of operation (see the enumerated list at the beginning of Section 9 for a listing of the operation types). Each of these sections displays the name of every operation of that type that is recorded in the database, regardless of whether the operation is queued, running, finished, or cancelled. Deleted operations do not appear.

The name of a given operation is actually the heading of a collapsed field that can be expanded by clicking on the small grey triangle next to the operation name. Expanding a section reveals a three-column table listing all the input and output files associated with the operation, along with a short description of the file contents and options for viewing or exporting the file. A link appears at the end of each table to export all of the files at once in a single compressed archive to your Desktop or Downloads folder.

\subsection{Microstructure Files}

Finished microstructure operations have either seven or eight associated files, each of which is briefly described here.

\begin{tabular}{l} 
V Cement152-Mortar \\
\begin{tabular}{|l|l|l|}
\hline \multicolumn{1}{|c|}{ File } & \multicolumn{1}{|c|}{ Description } & \multicolumn{1}{|c|}{ Content } \\
\hline ASTM-C109.gdg & Grading & Show Export \\
Cement152-Mortar.bias & One-pixel bias numbers & Show Export \\
Cement152-Mortar.img & Microstructure image & Show Export \\
Cement152-Mortar.img.in & Input to genmic & Show Export \\
Cement152-Mortar.img.out & Output from genmic & Show Export \\
Cement152-Mortar.img.struct & Particle SH coefficients & Show Export \\
Cement152-Mortar.pimg & Particle image file & Show Export \\
Cement152-Mortar.stt & Cement phase statistics & Show Export \\
\hline
\end{tabular} \\
Export all files for this operation in one zip file: Cement152-Mortar.zip \\
\hline
\end{tabular}


Grading: A text file containing the aggregate grading specified when creating a mortar or concrete. This entry will not be present for cement pastes. The contents of this file, and any other simple text file, can be displayed in a scrolling text box by clicking on the Show link at the right of the entry. It consists of three columns:

1. the common name of the sieve

2. the sieve opening size (in $\mathrm{mm}$ )

3. the mass fraction of aggregate retained in the sieve

The text box can be hidden again by clicking on the Hide link. Again, this or any other file on the My Files page can be exported to the Desktop or Downloads folder by clicking on the Export link.

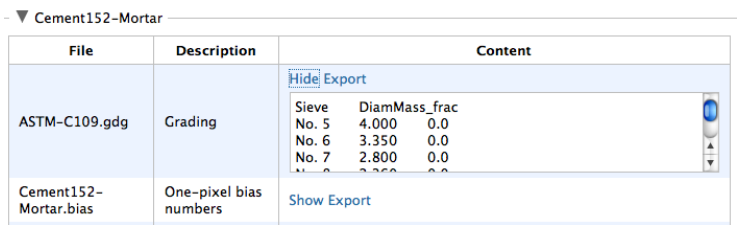

One-pixel bias numbers: Cement paste microstructures in VCCTL have a resolution of $1 \mu \mathrm{m}$, but a large number of cement particles are smaller than this minimum feature size. To include these particles in the virtual microstructure, they are represented as $1 \mu \mathrm{m}$ clusters of smaller particles. These clusters should have a higher surface area and, consequently, a greater rate of reaction with water than a single particle of the same size. The one-pixel bias numbers, calculated from knowledge of the cement particle size distribution in the submicrometer range, are included in this file as a triplet of numbers, one number on each row, for each phase in the initial microstructure. The triplet is formatted as follows:

- The first number in the triplet is always 0 , for reasons having to do with the legacy versions of the software.

- The second number in the triplet is the bias value, a decimal number typically near 1.0, but which may be significantly greater than 1.0 for fine cements, or slightly less than 1.0 for coarse cements.

- The third number in the triplet is the integer identification number of the phase. Appendix B lists the identification numbers for the phases.

Microstructure image: This file contains a listing of the phase occupying each voxel in the 3D virtual microstructure. The file has a five-line header with self-descriptive entries, followed by the integer phase identification number for each voxel in the grid, with one voxel per row. Ordinarily, the text version 
of this file will hold little interest to the user. Therefore, clicking on the Show link will cause a thumbnail color image of a 2D slice from the microstructure to display. Please refer to Section 10.2 for more information on working with these images.

Input to genmic: This is the input file read by the $\mathrm{C}$ program (genmic) that creates the virtual microstructure. It is difficult to read because it is not annotated in any way. More sense can be made of the input file by viewing it in conjunction with the output file, the latter which provides a running commentary on the types of input being read.

Output from genmic: This is the output file produced by the $C$ program (genmic) as it runs. This file ordinarily will be of little interest, but it can provide important clues to the cause of any failure of the program to execute properly. If genmic fails for some reason, an error message will typically appear near the end of the output file.

Particle SH coefficients: A listing of the coefficients in the spherical harmonic $(\mathrm{SH})$ expansion describing the shape of each particle in the microstructure. This file probably is of no interest to most users.

Particle image file: This file is a complement to the microstructure image file. Whereas the microstructure image associates a unique phase identification number to each voxel, the particle image file associates a particle number to each voxel. Specifically, a voxel is assigned a number $n$ if the phase at that voxel belongs to the nth particle in the microstructure. Porosity is assigned the number zero, and particles comprised of only one voxel also are assigned zero. This file is used internally by the $C$ program responsible for hydration operations (disrealnew) and should hold no interest for most users.

Cement phase statistics: A tabular display of the number of voxels assigned to each phase in the final microstructure, along with the corresponding mass fraction, volume fraction, and surface area fraction of that phase in the virtual microstructure. This file consists of two sections. The first eight lines of the file display the phase statistics on a total clinker solid basis. This section can be used to determine how closely the final microstructure reproduces the clinker phase fractions and $\mathrm{w} / \mathrm{b}$ ratio that were requested when the operation was launched. The remaining part of the file lists four values for each phase, in this order:

1. number of voxels assigned to that phase

2. number of voxel faces of that phase that are adjacent to porosity; this is the digitized analog of the surface area.

3. volume fraction of the phase in the microstructure, on a total volume basis (solids plus porosity) 
4. mass fraction of the phase in the microstructure, on a total mass basis (solids plus porosity)

\subsection{Displaying microstructure images}

As already described, microstructure images are displayed as color 2D slices of the 3D microstructure. VCCTL provides the ability to view the microstructure at different magnifications and from different perspectives, so we now devote attention to explaining these features.

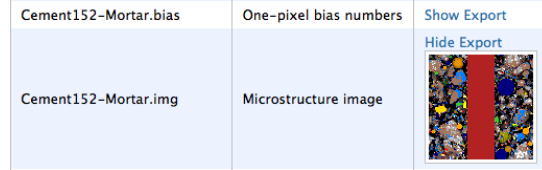

Clicking on the Show link for any microstructure image file displays a thumbnail image of a 2D cross section from the 3D virtual microstructure. Note that it may take several seconds for this image to display because it is being created at the time of the Show request.

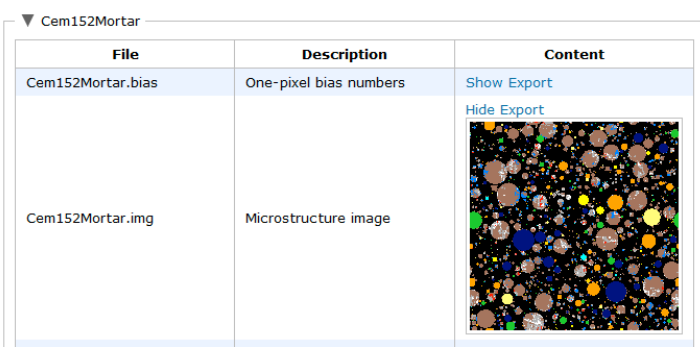

The thumbnail is a color image, with each color corresponding to a different phase in the mix. Once the thumbnail is displayed, it can be clicked with the mouse to open another page for more detailed examination and manipulation of the image. 


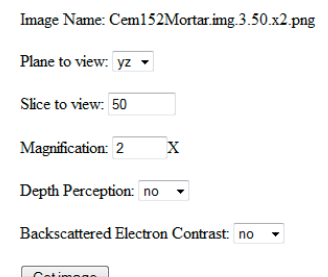

Getimage

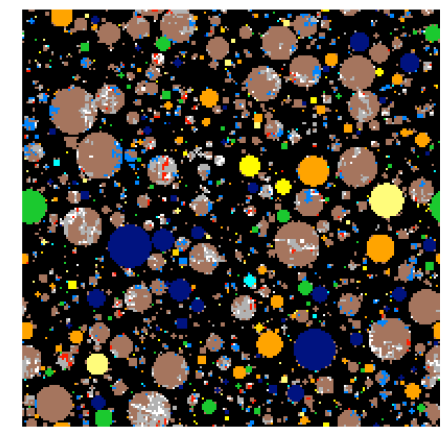

Plane to view: Choose one of three orthogonal directions from which to view the microstructure. The viewing planes are defined by the two Cartesian coordinate axes they contain (e.g., the $y z$-plane is the plane observed when viewing along the $x$-axis).

Slice to view: Changing this value enables the user to view the image at different locations along the axis of viewing. Valid values are in the interval $\left[0, L_{x}-1\right]$, where $L_{x}$ is the number of voxels in the direction being viewed. Values outside the valid interval are converted to the nearest value in the valid interval. For example, a slice value of -7 would be converted to 0 .

Magnification: A positive integer value of $M$ displays the image with a square of $M \times M$ image pixels corresponding to each voxel in the original image slice. Note: the magnification value must be a positive integer.

Depth Perception: Selecting yes from the pull-down menu will reveal the microstructure just below the surface of the current slice, much as if the porosity were filled with a murky liquid. 


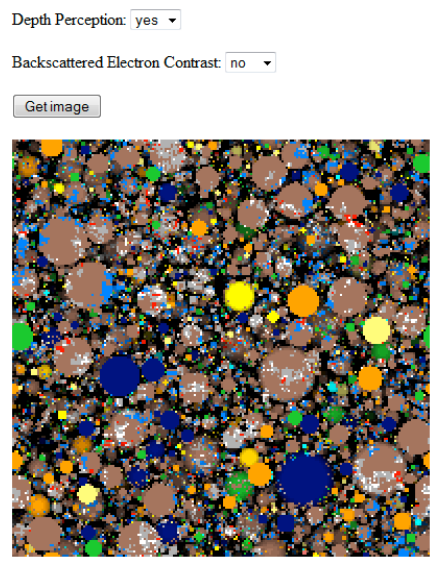

Backscattered Electron Contrast: Selecting yes from the pull-down menu will display a gray-scale image of the microstructure in which the relative brightness of each phase corresponds approximately to what would be observed in a backscattered electron image of a polished section using a scanning electron microscope.

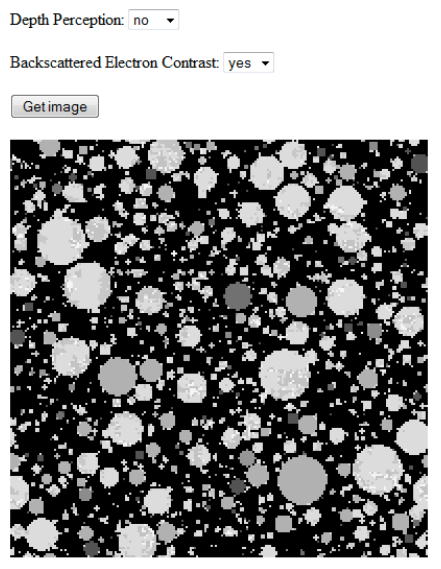

Once the viewing options are selected, click the Get image button or just press the Enter key on the keyboard to view the new image. Images previously viewed while this page has been open can be accessed by clicking the Back button on your browser.

Underneath the displayed image is a key showing the colors assigned to each phase in the microstructure. The color key is identical to that used to view cements on the Lab Materials page, described in Section 6.1.

\subsection{Aggregate Files}

Finished aggregate packing operations have seven associated files, each of which is briefly described here. 


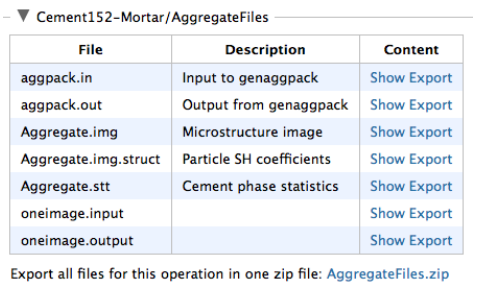

Input to genaggpack: This is the input file read by the $C$ program (genaggpack) that creates the virtual microstructure. It is difficult to read because it is not annotated in any way. More sense can be made of the input file by viewing it in conjunction with the output file, the latter which provides a running commentary on the types of input being read.

Output from genaggpack: This is the output file produced by the $\mathrm{C}$ program (genaggpack) as it runs, especially describing the nature of the input values that are read. Note: Ordinarily, the output file will be of little interest, but it can provide important clues to the cause of any failure of the program to execute properly. If genaggpack fails for some reason, an error message will typically appear near the end of the output file.

Microstructure image: This file contains a listing of the phase occupying each voxel in the 3D virtual microstructure. In contrast to cement paste microstructures, an aggregate packing has either two or three phases present. Porosity and aggregate are always present. In addition, if the resolution of the packing is sufficiently fine that the ITZ can be resolved, it will appear as a separate phase for easy visualization. Clicking on the Show link will cause a thumbnail color image of a 2D slice from the microstructure to display. Please refer to Section 10.2 for more information on working with these images.

Particle SH coefficients: A listing of the coefficients in the spherical harmonic (SH) expansion describing the shape of each particle in the packing. This file is of no interest to most users.

Phase statistics: A tabular display of the number of voxels assigned to each phase in the final packing. There is one line for porosity, one line for aggregate, and one line for ITZ. A value of zero for ITZ does not mean that there is no ITZ in the mortar or concrete, but instead means that the virtual packing was created at too coarse a resolution to include the ITZ in the image.

Besides these five files, a oneimage.input file and a oneimage. output file are also present in the table. These are used in the auxiliary program to render the aggregate packing image, and are of no interest to VCCTL users.

\subsection{Hydration Files}

Finished hydration operations often have a dozen or more associated files. 


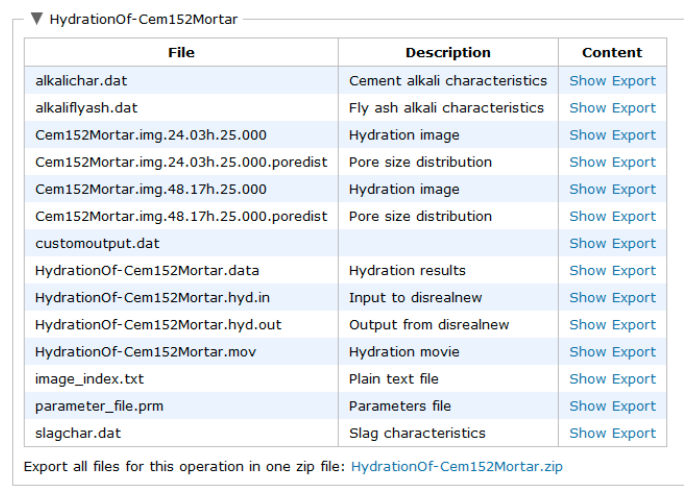

Cement alkali characteristics: Contains information on both the total and the readily-soluble potassium and sodium in the cement. The format and viewing of this file were discussed in Section 6.1.

Fly ash alkali characteristics: This file is required even if the material does not contain any fly ash. Its format is identical to that for the cement alkali characteristics file.

Hydration image: There will likely be ten or more of this type of file in the list. Each one is a saved 3D microstructure at a different time during hydration. The actual number of this type of file depends on the time interval that was specified between successive outputs and on the total simulated time. For example, if a user requests hydration for $28 \mathrm{~d}(672 \mathrm{~h})$ with microstructure output every $72 \mathrm{~h}$, there will be 10 hydration images $(72 \mathrm{~h}, 144 \mathrm{~h}, 216 \mathrm{~h}, 288 \mathrm{~h}, 360 \mathrm{~h}, 432 \mathrm{~h}$, $504 \mathrm{~h}, 576 \mathrm{~h}, 648 \mathrm{~h}$, and a final microstructure at $672 \mathrm{~h}$ when the run finishes). All but the final hydration image files have the following pattern for its name:

MicrostructureName.img.xxx.xxh.TT.Oyz

where

- MicrostructureName is the name given to the initial microstructure (i.e., the microstructure operation name).

- $\mathrm{xxx} \cdot \mathrm{xxh}$ is the number of hours (to the nearest $0.01 \mathrm{~h}$ ) of hydration to which this image corresponds. Note: The exact time at which the microstructure is output is as close to the desired time interval as possible, but the elapsed time of each hydration cycle is not constant and so output at the exact time interval requested is rarely possible.

- TT is the initial temperature used for the hydration operation $\left({ }^{\circ} \mathrm{C}\right)$.

- y is 0 if isothermal conditions were specified, or is 1 if adiabatic or semiadiabatic conditions were specified. 
- $\mathrm{z}$ is 0 if hydrating under saturated conditions, or is 1 if hydrating under sealed conditions.

As an example, a hydrated image name of MyMic.img.289.20h.25.000 means the original microstructure is named MyMic.img, the microstructure was output at $289.20 \mathrm{~h}$ of hydration time, the initial temperature was $25^{\circ} \mathrm{C}$, and isothermal and saturated conditions were used.

The exception to this naming pattern is the final image that is written when the hydration operation terminates. This final image has the pattern

HydrationName.img. TT. Oyz

where HydrationName is the name of the hydration operation, and TT, y, and z have the same meaning as before.

Clicking the Show link for any of the hydration images will cause a thumbnail color image of a $2 \mathrm{D}$ slice from the microstructure to display. Please refer to Section 10.2 for more information on working with these images.

Hydration movie: A file of this type (with extension .mov) will be present if and only if the relevant option was selected when the hydration simulation was launched. Clicking the Show link will display a thumbnail animation of the progress of hydration of a 2D slice from the microstructure. Clicking on the thumbnail will produce a larger image that can be viewed at different magnifications and different viewing speeds.

Pore size distribution: A file with extension poredist is created with every image file. Clicking on the Show link will display a bar chart representation of the capillary pore size distribution of the simulated microstructure, plotted as a differential distribution. Unlike images, the thumbnail image cannot be expanded and manipulated.

Input to disrealnew: This is the input file read by the $C$ program (disrealnew) that performs the virtual hydration operation. It is difficult to read because it is not annotated in any way. More sense can be made of the input file by viewing it in conjunction with the output file, the latter which provides a running commentary on the types of input being read.

Output from disrealnew: This is the output file produced by the $C$ program (disrealnew) as it runs, especially describing the nature of the input values that are read. Note: Ordinarily, the output file will be of little interest, but it can provide important clues to the cause of any failure of the program to execute properly. If disrealnew fails for some reason, an error message will typically appear near the end of the output file.

Image_index.txt: This is a two-column text file. The left column gives the times at which the hydration images were output, and the right column contains the full path name to the hydration image in question. Ordinarily, this file will be of no interest to most users, because any or all the images can be exported to the Desktop or Downloads folder by clicking on the Export link. 
Parameters file: A listing of the several dozen parameters used by the $C$ program (disrealnew) in its execution. These parameters cannot be modified by the user.

Slag characteristics: Contains information about the physical and chemical characteristics of the slag, and the slag hydration products, used in the microstructure. This file must be input to the hydration operation even if no slag is present in the microstructure. The format and viewing of this file were discussed in Section 6.2.

Hydration results: A large tabular display of the values of the 63 hydration properties at each computational cycle. This file has 63 columns and several hundred rows, making it difficult to view in the small text box that is provided when the Show button is clicked. It is much more convenient to export the file and then import it into a spreadsheet application. Also, recall that any one or more of the properties in this file can be simultaneously plotted on the Measurements page of VCCTL. The meaning of each of the 63 columns in the hydration results file is provided in Appendix C.

\subsection{Mechanical Properties Files}

Finished mechanical properties operations have five associated files, each of which is briefly described here.

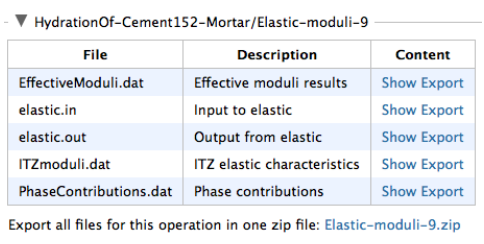

Input to elastic: This is the input file read by the $C$ program (elastic) that performs both the finite element calculations of the effective elastic moduli of cement paste and, for mortar or concrete, the effective medium theory calculations of the effective moduli and compressive strength of the material. This file has only a few lines, but it is not easy to understand because it is not annotated. More sense can be made of the input file by viewing it in conjunction with the output file, the latter which provides a running commentary on the types of input being read.

Output from elastic: This is the output file produced by the C program (elastic) as it runs, especially describing the nature of the input values that are read. Note: Ordinarily, the output file will be of little interest, but it can provide important clues to the cause of any failure of the program to execute properly. If elastic fails for some reason, an error message will typically appear near the end of the output file. 
Effective moduli results: This file is displayed in the first text box on the Measurements page when a mechanical property operation is finished. Its format is described in Section 8.2.

Phase contributions: This file is displayed in the second text box on the Measurements page when a mechanical property operation is finished. Its format is described in Section 8.2.

ITZ elastic characteristics: This file is present only if aggregate has been included in the mix (i.e., only for a mortar or concrete). It is displayed in the third text box on the Measurements page when a mechanical property operation is finished. Its format is described in Section 8.2.

\subsection{Transport Properties Files}

Finished transport properties operations have four associated files, each of which is briefly described here.

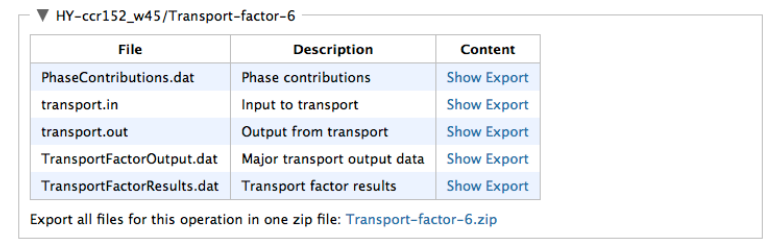

Phase contributions: This file is displayed in the second text box on the Measurements page when a transport property operation is finished. Its format is described in Section 8.2.

Input to transport: This is the input file read by the $C$ program (dc3d) that performs both the finite difference calculations of the effective relative diffusion coefficients and formation factor of the given microstructure image. This file has only a few lines, but it is not easy to understand because it is not annotated. More sense can be made of the input file by viewing it in conjunction with the output file, the latter which provides a running commentary on the types of input being read.

Output from transport: This is the output file produced by the C program (dc3d) as it runs, especially describing the nature of the input values that are read. Note: Ordinarily, the output file will be of little interest, but it can provide important clues to the cause of any failure of the program to execute properly. If $\mathrm{dc} 3 \mathrm{~d}$ fails for some reason, an error message will typically appear near the end of the output file.

Major transport output data: This file contains rather verbose output from the dc3d program, including the status of the conjugate gradient relaxation method [Garboczi98] at the end of each iteration. Ordinarily, this file will hold no interest for the user. 
Transport factor results: This file is displayed in the first text box on the Measurements page when a transport property operation is finished. Its format is described in Section 8.2.

\section{Logout}

To gracefully log out of the VCCTL system, click on the Logout tab in the main menu bar. This logs you out immediately and also takes you to the original login page for the system, where you can switch to a new user.

Note: If you wish to shut down VCCTL and close the browser, as explained in Section 3, you must also

- wait for all running operations to finish, or cancel all running operations,

- close the web browser, and then

- open the Java console window and press $C t r l-C$. After a brief pause, you will be asked, Terminate batch job $(Y / N)$ ?. Type $y$ and press the Enter button. The Java console window will close. 


\section{Appendices}

\section{A Preparing and Uploading New Cement Data}

VCCTL comes packaged with an executable file called process Image J.exe that can be run from the command line to package the data for a new cement into the kinds of data files that ultimately will be needed to create the zipped archive needed to upload the cement into VCCTL. This program is designed to take just the segmented and indexed image file and create nearly all the files necessary to upload a cement into VCCTL 9. The program runs from the command line in an interactive way, prompting the user for responses that it needs to create the text files.

\section{A.1 Getting Ready}

1. Make sure that the processImageJ.exe executable file is somewhere in your PATH environment, or else be prepared to type in the full path name to it when you want to run it.

2. You must have ImageMagick installed on your computer. It can be downloaded freely at

http://www.imagemagick.org/script/binary-releases.php\#windows

Just download and run the executable from the website to begin the installation, and choose all the defaults during the installation.

3. Create a folder and put your segmented, indexed image file in the folder. Note: the indexed image file must have the extension .txt.

4. For the purposes of these instructions, we will call the folder NewCement, and we will call the image file NewCementIndexedImage.txt.

5. Decide the name you want this cement to have in VCCTL. For the purposes of these instructions, we will use the name MyNewCement

6. To make the best use of this program, you should have the following data ready when you run it, because the program will prompt you for these data. You don't need to have them, but you will have to go back and modify the individual files later if not.

(a) The spatial resolution of the image, in units of $\mu \mathrm{m} /$ pixel. This is important because it sets the scale for all the correlation function kernels that will get created. Prior experience indicates that an incorrect scaling will not dramatically affect VCCTL results, but it can affect the fidelity with which VCCTL microstructures will mimic the real microstructure. 
(b) The mass fraction (not percentage) of gypsum, hemihydrate, and anhydrite in the cement, on a total solids basis.

(c) $\mathrm{Na}_{2} \mathrm{O} \%$ by mass

(d) $\mathrm{K}_{2} \mathrm{O} \%$ by mass

(e) Readily soluble $\mathrm{Na}_{2} \mathrm{O} \%$ by mass (less than or equal to total $\mathrm{Na}_{2} \mathrm{O} \%$ by mass)

(f) Readily soluble $\mathrm{K}_{2} \mathrm{O} \%$ by mass (less than or equal to total $\mathrm{K}_{2} \mathrm{O} \%$ by mass)

(g) Percent of total $\mathrm{Na}_{2} \mathrm{O}$ added as $\mathrm{NaOH}$ to mix water (usually zero)

(h) Percent of total $\mathrm{K}_{2} \mathrm{O}$ added as $\mathrm{NaOH}$ to mix water (usually zero)

(i) A descriptive name for the cement (several words)

(j) Several words describing the source of the cement.

(k) Several words indicating the date when cement was characterized (e.g., April 2014)

(l) Surface area of the cement, in units of $\mathrm{m}^{2} / \mathrm{kg}$

(m) How the surface area was measured (e.g., Blaine, BET, particle size distribution)

(n) Knowledge of the indexing of the image (i.e., which phase is associated with which index in the image file).

\section{A.2 Running processImageJ}

1. Drag the NewCement folder to your Desktop.

2. Open a command window.

3. Type cd Desktop\NewCement.

4. Type FULL_PATH_TO_VCCTL_FOLDER \bin $\backslash$ processImageJ . exe and press Enter, where FULL_PATH_TO_VCCTL_FOLDER is the location of the VCCTL install folder

5. Answer each of the questions in turn, based on the information compiled in the previous section. The necessary files will be created in the NewCement directory.

\section{A.3 What To Do Next}

1. If you have the cement particle size distribution in the acceptable format, give it the same root name as all the other newly created files in the NewCement folder, using the extension . psd, and place it in the NewCement directory. The correct format is an ASCII text files with two columns separated by either a tab or space: 
(a) The particle diameter in $\mu \mathrm{m}$ units

(b) The differential mass fraction (not percent) for each size.

If you don't have the particle size distribution ready, you can add it later within the VCCTL software.

2. Optionally, if you have quantitative X-ray diffraction data available in ASCII text form (format is unimportant, usually some kind of tabular format), give it the same root name as all the other newly created files, using the extension .xrd, and place it in the NewCement directory with the other files. If you don't have the particle size distribution ready, you can add it later within the VCCTL software.

3. Collect all the newly created files in a single zip archive.

4. When you're ready, you can upload this zip file on the Lab Materials page of VCCTL.

5. After the upload has completed, the segmented image may not appear, but the other fields on the Lab Materials page should be updated as expected. Make sure to save the cement using the Save As button, and give the cement the same name as the root name of all the files created by process Image J.

6. The image of the cement should also appear the next time you visit the page and examine the new cement. 
B INTEGER ID NUMBERS OF VCCTL PHASES

\section{B Integer ID Numbers of VCCTL Phases}

This appendix gives the integer identification number and the short display name that is used in VCCTL for each phase that is recognized in the system.

\begin{tabular}{|c|c|c|}
\hline Phase Name & Display Name & ID \\
\hline Saturated porosity & POROSITY & 0 \\
\hline $\mathrm{C}_{3} \mathrm{~S}$ (alite) & C3S & 1 \\
\hline $\mathrm{C}_{2} \mathrm{~S}$ (belite) & $\mathrm{C} 2 \mathrm{~S}$ & 2 \\
\hline $\mathrm{C}_{3} \mathrm{~A}$ (cubic form) & $\mathrm{C} 3 \mathrm{~A}$ & 3 \\
\hline $\mathrm{C}_{4} \mathrm{AF}$ & $\mathrm{C} 4 \mathrm{AF}$ & 4 \\
\hline $\mathrm{K}_{2} \mathrm{SO}_{4}$ (arcanite) & $\mathrm{K} 2 \mathrm{SO} 4$ & 5 \\
\hline $\mathrm{Na}_{2} \mathrm{SO}_{4}$ (thenardite) & $\mathrm{NA} 2 \mathrm{SO} 4$ & 6 \\
\hline Gypsum $\left(\mathrm{CaSO}_{4} \cdot 2 \mathrm{H}_{2} \mathrm{O}\right)$ & GYPSUM & 7 \\
\hline Bassanite $\left(\mathrm{CaSO}_{4} \cdot 0.5 \mathrm{H}_{2} \mathrm{O}\right)$ & HEMIHYD & 8 \\
\hline Anhydrite $\left(\mathrm{CaSO}_{4}\right)$ & ANHYDRITE & 9 \\
\hline Silica fume & SFUME & 10 \\
\hline Inert filler & INERT & 11 \\
\hline Slag & SLAG & 12 \\
\hline Aggregate & INERTAGG & 13 \\
\hline Aluminosilicate glass in fly ash & ASG & 14 \\
\hline Calcium aluminosilicate glass in fly ash & CAS2 & 15 \\
\hline Amorphous silica & AMSIL & 16 \\
\hline $\mathrm{C}_{3} \mathrm{~A}$ in fly ash & FAC3A & 17 \\
\hline Portlandite $(\mathrm{CH})$ & $\mathrm{CH}$ & 19 \\
\hline C-S-H gel & $\mathrm{CSH}$ & 20 \\
\hline Hydrogarnet $\left(\mathrm{C}_{3} \mathrm{AH}_{6}\right)$ & C3AH6 & 21 \\
\hline $\mathrm{AFt}$ (generic ettringite) & ETTR & 22 \\
\hline AFt with Fe substitution & ETTRC4AF & 23 \\
\hline AFm (generic monosulfate) & AFM & 24 \\
\hline $\mathrm{Fe}(\mathrm{OH})_{3}$ & FH3 & 25 \\
\hline $\mathrm{C}-\mathrm{S}-\mathrm{H}$ gel with low $\mathrm{Ca} / \mathrm{Si}$ & POZZCSH & 26 \\
\hline C-S-H from slag reactions & SLAGCSH & 27 \\
\hline $\mathrm{CaCl}_{2}$ & CACL2 & 28 \\
\hline Friedel's salt $\left(\mathrm{C}_{3} \mathrm{~A} \cdot \mathrm{CaCl}_{2} \cdot 10 \mathrm{H}_{2} \mathrm{O}\right)$ & FRIEDEL & 29 \\
\hline Strätlingite $\left(\mathrm{C}_{2} \mathrm{ASH}_{8}\right)$ & STRAT & 30 \\
\hline Secondary gypsum & GYPSUMS & 31 \\
\hline $\mathrm{CaCO}_{3}$ (generic limestone) & $\mathrm{CACO} 3$ & 33 \\
\hline Calcium monocarboaluminate & AFMC & 34 \\
\hline Brucite $\left(\mathrm{Mg}(\mathrm{OH})_{2}\right)$ & BRUCITE & 35 \\
\hline $\mathrm{MgSO}_{4}$ & MS & 36 \\
\hline Free lime $(\mathrm{CaO})$ & FREELIME & 37 \\
\hline $\mathrm{C}_{3} \mathrm{~A}$ (orthorhombic form) & OC3A & 38 \\
\hline Dried porosity & DRIEDP & 53 \\
\hline Self-desiccated porosity & EMPTYP & 55 \\
\hline
\end{tabular}




\section{Contents of Hydration Results File}

This appendix provides a brief description of each column in the hydration results file (ending in .data; see Section 10.4 for more information).

\begin{tabular}{|c|c|c|}
\hline Column & Heading & Description \\
\hline 1 & Cycle & computational cycle \\
\hline 2 & time (h) & hydration time (h) \\
\hline 3 & Alpha_mass & $\begin{array}{l}\text { degree of hydration on a cement mass ba- } \\
\text { sis }\end{array}$ \\
\hline 4 & Alpha_fa_mass & $\begin{array}{l}\text { degree of hydration of fly ash on a fly ash } \\
\text { mass basis }\end{array}$ \\
\hline 5 & Heat (kJ/kg_solid) & $\begin{array}{l}\text { heat liberated ( }>0 \text { for exothermic, } \mathrm{kJ} / \mathrm{kg} \\
\text { of solids) }\end{array}$ \\
\hline 6 & Temperature(C) & system temperature $\left({ }^{\circ} \mathrm{C}\right)$ \\
\hline 7 & Gsratio & paste gel-space ratio (see [22]) \\
\hline 8 & Wno $(g / g)$ & $\begin{array}{l}\text { non-evaporable water ( } \mathrm{g} \text { water/ } \mathrm{g} \text { ce- } \\
\text { ment) on an unignited cement mass basis }\end{array}$ \\
\hline 9 & $\operatorname{Wni}(\mathrm{g} / \mathrm{g})$ & $\begin{array}{l}\text { non-evaporable water content (g water/ } \\
\text { g cement) on an ignited cement mass }\end{array}$ \\
\hline 10 & ChemShrink(mL/g) & chemical shrinkage (mL/g of cement) \\
\hline 11 & $\mathrm{pH}$ & $\begin{array}{l}\text { estimated } \mathrm{pH} \text { of the capillary pore solu- } \\
\text { tion }\end{array}$ \\
\hline 12 & Conductivity & $\begin{array}{l}\text { estimated DC conductivity of the pore } \\
\text { solution }(S / m)\end{array}$ \\
\hline 13 & {$[\mathrm{Na}+](\mathrm{M})$} & estimated $\mathrm{Na}^{+}$in solution $(\mathrm{mol} / \mathrm{L})$ \\
\hline 14 & {$[\mathrm{~K}+](\mathrm{M})$} & estimated $\mathrm{K}^{+}$in solution (mol/L) \\
\hline 15 & {$[\mathrm{Ca}++](\mathrm{M})$} & estimated $\mathrm{Ca}^{2+}$ in solution $(\mathrm{mol} / \mathrm{L})$ \\
\hline 16 & [SO4--] (M) & estimated $\mathrm{SO}_{4}^{2-}$ in solution $(\mathrm{mol} / \mathrm{L})$ \\
\hline 17 & $\{\mathrm{~K}+\}$ & estimated molal activity of $\mathrm{K}^{+}$in solution \\
\hline 18 & $\{\mathrm{Ca}++\}$ & $\begin{array}{l}\text { estimated molal activity of } \mathrm{Ca}^{2+} \text { in solu- } \\
\text { tion }\end{array}$ \\
\hline 19 & $\{\mathrm{OH}-\}$ & $\begin{array}{l}\text { estimated molal activity of } \mathrm{OH}^{-} \text {in solu- } \\
\text { tion }\end{array}$ \\
\hline 20 & $\{\mathrm{SO} 4--\}$ & $\begin{array}{l}\text { estimated molal activity of } \mathrm{SO}_{4}^{2-} \text { in solu- } \\
\text { tion }\end{array}$ \\
\hline 21 & Vfpore & Volume fraction of capillary pores \\
\hline
\end{tabular}


C CONTENTS OF HYDRATION RESULTS FILE

\begin{tabular}{|c|c|c|}
\hline Column & Heading & Description \\
\hline 22 & Poreconnx & $\begin{array}{l}\text { Fraction of capillary pore volume that forms a } \\
\text { percolated network in the } x \text { direction }\end{array}$ \\
\hline 23 & Poreconny & $\begin{array}{l}\text { Fraction of capillary pore volume that forms a } \\
\text { percolated network in the } y \text { direction }\end{array}$ \\
\hline 24 & Poreconnz & $\begin{array}{l}\text { Fraction of capillary pore volume that forms a } \\
\text { percolated network in the } z \text { direction }\end{array}$ \\
\hline 25 & Poreconnave & Arithmetic mean of columns 22-24 \\
\hline 26 & Solidconnx & $\begin{array}{l}\text { Fraction of solids that form a percolated network } \\
\text { in the } x \text { direction }\end{array}$ \\
\hline 27 & Solidconny & $\begin{array}{l}\text { Fraction of solids that form a percolated network } \\
\text { in the } y \text { direction }\end{array}$ \\
\hline 28 & Solidconnz & $\begin{array}{l}\text { Fraction of solids that form a percolated network } \\
\text { in the } z \text { direction }\end{array}$ \\
\hline 29 & Solidconnave & Arithmetic mean of the columns $26-28$ \\
\hline $30-63$ & Vfxxx & $\begin{array}{l}\text { Volume fraction of phase } \mathrm{xxx} \text { on a system volume } \\
\text { basis, where xxx is the display name of the phase } \\
\text { given in Appendix I. }\end{array}$ \\
\hline
\end{tabular}




\section{References}

[1] Dale P. Bentz. Three-dimensional computer simulation of cement hydration and microstructure development. J. Am. Ceram. Soc., 80(1):3-21, 1997. $1,7.1,7.3$

[2] Dale P. Bentz. CEMHYD3D: A three-dimensional cement hydration and microstructure development modeling package. Version 3.0. NISTIR 7232, U.S. Department of Commerce, June 2005. Available at http://ciks.cbt.nist.gov/monograph. 1, 7.1, 7.3

[3] Dale P. Bentz and J. T. Conway. Computer modeling of the replacement of 'coarse' cement particles by inert fillers in low w/c ratio concretes: Hydration and strength. Cem. Concr. Res., 31:503-506, 2002. 1

[4] Dale P. Bentz, X. Feng, Claus J. Haecker, and Paul E. Stutzman. Analysis of CCRL proficiency cements 135 and 136 using CEMHYD3D. NISTIR 6545, U.S. Department of Commerce, August 2000. Available online at the NIST electronic monograph, http:/ / ciks.cbt.nist.gov/monograph, Part I, Chapter 4, Section 3. 1

[5] Dale P. Bentz and Edward J. Garboczi. Simulation studies of the effects of mineral admixtures on the cement paste-aggregate interfacial zone. ACI Mater. J., 88:518-529, 1991. 1

[6] Dale P. Bentz and Edward J. Garboczi. Computer modelling of interfacial transition zone microstructure and properties. In M. G. Alexander, G. Arliguie, G. Ballivy, A. Bentur, and J. Marchand, editors, Engineering and Transport Properties of the Interfacial Transition Zone in Cementitious Composites, pages 349-385. RILEM, Paris, FRANCE, 1999. 7.2, 8.2

[7] Dale P. Bentz, Edward J. Garboczi, Claus J. Haecker, and Ole M. Jensen. Effects of cement particle size distribution on performance properties of cement-based materials. Cem. Concr. Res., 29(10):1663-1671, 1999. 1

[8] Dale P. Bentz and Claus J. Haecker. An argument for using coarse cements in high performance concretes. Cem. Concr. Res., 29:615-618, 1999. 1

[9] Dale P. Bentz, Ole M. Jensen, K. K. Hansen, J. F. Olesen, H. Stang, and Claus J. Haecker. Influence of cement particle size distribution on early age autogenous strains and stresses in cement-based materials. J. Am. Ceram. Soc., 84(1):129-135, 2000. 1

[10] Dale P. Bentz and Paul E. Stutzman. SEM analysis and computer modelling of hydration of Portland cement particles. In S. M. DeHayes and D. Stark, editors, Petrography of Cementitious Materials, pages 60-73. American Society for Testing and Materials, Philadelphia, PA, 1994. 6.1 
[11] Dale P. Bentz, Paul E. Stutzman, Claus J. Haecker, and S. Remond. SEM/X-ray imaging of cement-based materials. In H. S. Pietersen, J. A. Larbia, and H. H. A. Janssen, editors, Proceedings of the 7th Euroseminar on Microscopy Applied to building Materials, pages 457-466. Delft University of Technology, 1999. 6.1

[12] Jeffrey W. Bullard and Edward J. Garboczi. A model investigation of the influence of particle shape on portland cement hydration. Cem. Concr. Res., 36(6):1007-1015, 2006. 1

[13] Jeffrey W. Bullard and Paul E. Stutzman. Analysis of CCRL Portland cement proficiency samples number 151 and number 152 using the virtual cement and concrete reference laboratory. Cem. Concr. Res., 36:1548-1555, 2006. 1

[14] X. Feng, Edward J. Garboczi, Dale P. Bentz, Paul E. Stutzman, and Thomas O. Mason. Estimation of the degree of hydration of blended cement pastes by a scanning electron microscope point-counting procedure. Cem. Concr. Res., 34(10):1787-1793, 2004. 6.3

[15] Edward J. Garboczi. Finite element and finite difference programs for computing the linear electric and elastic properties of digital images of random materials. NISTIR 6269, U.S. Department of Commerce, December 1998. 1, 8.2

[16] Edward J. Garboczi. Three-dimensional mathematical analysis of particle shape using X-ray tomography and spherical harmonics: Application to aggregates used in concrete. Cem. Concr. Res., 32(10):1621-1638, 2002. 6.5

[17] Edward J. Garboczi and Dale P. Bentz. The effect of statistical fluctuation, finite size error, and digital resolution on the phase percolation and transport properties of the NIST cement hydration model. Cem. Concr. Res., 31:1501-1514, 2001. 7.1

[18] Edward J. Garboczi, Dale P. Bentz, and Nicos S. Martys. Digital images and computer modelling. In P-z. Wong, editor, Methods in the Physics of Porous Media, number 35 in Experimental methods in the Physical Sciences, pages 1-41. Academic Press, San Diego, CA, 1999. 7.1

[19] Edward J. Garboczi and James G. Berryman. Elastic moduli of a material containing composite inclusions: Effective medium theory and finite element computations. Mech. Mater., 3e:455-470, 2001. 8.2

[20] Edward J. Garboczi and Jeffrey W. Bullard. Shape analysis of a reference cement. Cem. Concr. Res., 34:1933-1937, 2004. 7.2

[21] A. M. Neville. Properties of Concrete. Prentice-Hall, Harlow, England, 1995. 8.2 
[22] Hal F. W. Taylor. Cement Chemistry. Thomas Telford, London, second edition, 1997. C 